\title{
Functional interdependence of BRD4 and DOT1L in MLL leukemia
}

Omer Gilan*1,2, Enid Y. N. Lam*1,2, Isabelle Becher ${ }^{3}$, Dave Lugo ${ }^{4}$, Ester Cannizzaro $^{5,}$, Gerard Joberty ${ }^{3}$, Aoife Ward ${ }^{3}$, Meike Wiese ${ }^{5}$, Chun Yew Fong, 2,6, Sarah Ftouni ${ }^{1}$, Dean Tyler ${ }^{1,2}$, Kym Stanley ${ }^{1}$, Laura MacPherson ${ }^{1}$, Chen-Fang Weng ${ }^{1}$, Yih-Chih Chan ${ }^{1}$, Margherita Ghisi ${ }^{1}$, David Smil ${ }^{7}$, Christopher Carpenter ${ }^{8}$, Peter Brown7, Neil Garton4, Marnie E. Blewitt9,10, Andrew J. Bannister5, Tony Kouzarides $^{5}$, Brian J. P. Huntly ${ }^{11,12}$, Ricky W. Johnstone ${ }^{1,2}$, Gerard Drewes ${ }^{3}$, SarahJane Dawson ${ }^{1,2,14}$, Cheryl H. Arrowsmith ${ }^{7,13}$, Paola Grandi ${ }^{3}$, Rab K. Prinjha ${ }^{4}$, Mark A. Dawson ${ }^{1,2,6,14}$

${ }^{1}$ Cancer Research Division, Peter MacCallum Cancer Centre, Melbourne, Victoria, Australia, ${ }^{2}$ Sir Peter MacCallum Department of Oncology, The University of Melbourne, Melbourne, Victoria, Australia, ${ }^{3}$ Cellzome GmbH, Molecular Discovery Research, GlaxoSmithKline, Heidelberg, Germany, ${ }^{4}$ Epinova DPU, Immuno-Inflammation Therapy Area Unit, GlaxoSmithKline, Stevenage, United Kingdom, 5The Gurdon Institute, University of Cambridge, Cambridge, United Kingdom, ${ }^{6}$ Department of Haematology, Peter MacCallum Cancer Centre, Melbourne, Victoria, Australia, ${ }^{7}$ Structural Genomics Consortium, University of Toronto, Toronto, Ontario, Canada, ${ }^{8}$ Cancer Epigenetics DPU, GlaxoSmithkline, USA, ${ }^{9}$ The Walter and Eliza Hall Institute of Medical Research, Parkville, Victoria, Australia ${ }^{10}$ Department of Medical Biology, The University of Melbourne, Victoria, Australia, ${ }^{11}$ Department of Haematology, Cambridge Institute for Medical Research, Cambridge, United Kingdom ${ }^{12}$ Cambridge Stem Cell Institute, Cambridge, United Kingdom, 13Princess Margaret Cancer Centre, Toronto, Ontario, Canada, ${ }^{14}$ Centre for Cancer Research, The University of Melbourne, Melbourne, Victoria, Australia.

* These authors contributed equally to this manuscript

\section{Corresponding Author:}

Associate Professor Mark A. Dawson

Cancer Epigenetics Laboratory and Department of Haematology, Peter MacCallum Cancer Centre, St. Andrews Place, East Melbourne, Australia, 3002

Email - mark.dawson@petermac.org

Phone: +61 39656 3728; Fax: +61 396561411 


\section{ABSTRACT}

Targeted therapies against disrupter of telomeric silencing 1-like (DOT1L) and bromodomain containing protein 4 (BRD4) are currently being evaluated in clinical trials. However, the mechanisms by which BRD4 and DOT1L regulate leukemogenic transcription programs remain unclear. Using quantitative proteomics, chemoproteomics and biochemical fractionation we find that native BRD4 and DOT1L exist in separate protein complexes. Genetic disruption or small molecule inhibition of BRD4 and DOT1L shows marked synergistic activity against MLL leukemia cell lines, primary human leukemia cells and mouse leukemia models. Mechanistically, we find a previously unrecognized functional collaboration between DOT1L and BRD4 that is especially important at highly transcribed genes in close proximity to superenhancers. DOT1L via H3K79me2 facilitates the deposition of histone $\mathrm{H} 4$ acetylation, which in turn regulates the binding of BRD4 to chromatin. These data provide novel insights into the regulation of transcription and specify a molecular framework for therapeutic intervention in this poor prognostic disease. 


\section{INTRODUCTION}

The established principle in oncology is that cancer is a disease, which is initiated and maintained by somatic alterations in our genome. Recent advances in DNA sequencing technologies have reaffirmed this fact and greatly facilitated our molecular understanding of the genetic basis for various malignancies ${ }^{1}$. The emerging challenge is to now decipher how these genomic alterations culminate in malignant transformation and how they can be targeted for therapeutic gain. A central theme emerging from the cancer genome data is recurrent mutations in epigenetic regulators ${ }^{2}$. In fact it is now apparent that mutations and/or translocations of epigenetic regulators are some of the most prevalent abnormalities observed in hematopoietic malignancies ${ }^{3,4}$. The realization that epigenetic regulators are widely implicated in the initiation and maintenance of cancer has fuelled substantial interest in identifying targeted therapies that alter malignant transcription programs and offer new opportunities for therapeutic intervention in these malignancies. Importantly several novel epigenetic therapies have now transitioned into the clinical arena where they are being assessed in a range of tumours ${ }^{2,5,6}$. Whilst combination therapy is the cornerstone of cancer management and is also the future for emerging epigenetic therapies, several lines of evidence caution against an empirical approach to combination epigenetic therapy. Without a sound molecular basis additional benefits are often not conferred by these combinations and in some cases the combination of epigenetic therapies have resulted in significant functional antagonism ${ }^{7-9}$. Therefore, preferred combinations should be guided by a thorough preclinical evaluation based on a comprehensive understanding of the molecular pathogenesis of the disease.

The mixed lineage leukemia (MLL) gene codes for a histone methyltransferase that plays an integral role in normal embryogenesis and the maintenance of both fetal and adult haematopoiesis ${ }^{10-12}$. Importantly, chromosomal translocations involving this essential epigenetic regulator accounts for over $70 \%$ of infantile leukemia and up to $10 \%$ of adult leukemia ${ }^{13}$. These MLL translocated leukemias follow an aggressive clinical course with a poor response to conventional 
treatment, highlighting the urgent need for better therapies in this group of diseases $^{13}$. The poor prognosis conferred by MLL fusion protein (MLL-FP) leukemia has attracted significant research attention with the aim of studying transcriptional dysregulation to identify new therapeutic opportunities ${ }^{13}$. Thus far, several small molecules have been developed to target various components of the transcription machinery co-opted by MLL-FP14-17. Many of these targeted therapies have shown pre-clinical promise and are currently being evaluated in clinical trials. Despite this progress, our understanding of the molecular events that govern leukemogenic transcription programs driven by MLL-FP remains incomplete.

Over 60 different $M L L$-translocation partners have been identified and many of these have been shown to be members of multi-subunit protein complexes that regulate transcription. Indeed, a fundamental abnormality in transcriptional elongation appears to underpin the molecular pathogenesis of MLL translocated leukemias ${ }^{18}$. Recent studies have suggested that the functional integrity of two major protein complexes: the super-elongation complex (SEC) and the disruptor of telomeric silencing complex (DOT1Lc), is critical for malignant transformation by MLL-FP ${ }^{18}$. Whilst there is a general consensus that the various members of these complexes play an integral role in the pathogenesis of MLL-FP leukemia, there is significant ambiguity related to the composition of these complexes and the mechanisms of transformation.

We set out to understand if BRD4 and DOT1L were in separate nuclear complexes and if they function in a mutually exclusive manner to regulate the malignant transcription programs that underpin MLL-FP leukemia. Taken together our findings have established that BRD4 and DOT1L mainly reside in distinct protein complexes. Whilst these separate complexes regulate discrete transcription programs in MLL-FP leukemia, there is significant co-operation and interdependency at a subset of genes, which are in close proximity to superenhancers and critical to the molecular pathogenesis of MLL-FP leukemia. 
At these loci we find DOT1L via H3K79me2, facilitates the recruitment of EP300 to acetylate Histone $\mathrm{H} 4$, which in turn regulates the binding of BRD4 to chromatin and the subsequent transcriptional output of these genes. Dual targeting of these essential transcriptional regulators leads to a profound suppression of transcription at these genes, which are important to the maintenance of MLL-FP leukemia thus providing a molecular rationale for future combination therapies.

\section{RESULTS}

\section{BRD4 and DOT1L are in distinct macromolecular complexes}

Previous studies have established that the functional integrity of the transcription elongation complexes known to contain BRD4 or DOT1L are critical to the initiation and maintenance of MLL-FP leukemia ${ }^{14,19-25}$. Nonetheless, a significant unresolved issue of contention is whether BRD4 and its associated positive transcription elongation factor $b$ complex (PTEFb), composed of CyclinT1-2 and cyclin-dependent kinase 9 (CDK9), are in shared or separate complexes with the histone methyltransferase DOT1L. Methodological differences are likely to have contributed to this controversy, as virtually all of the studies performed to date have employed either transient or stable expression of epitope-tagged proteins for biochemical and proteomic analyses ${ }^{19-}$ 25. We initially sought to address this issue using quantitative mass spectrometry and specific antibodies against endogenous proteins. These data clearly demonstrate that BRD4 associates with CDK9 as part of the PTEFb and other members of the previously identified superelongation complex (SEC) 21,25. Importantly our data suggest that BRD4 and PTEFb are found separately to DOT1L in various haematopoietic cells that contain wild-type MLL or those that also harbour an MLL-FP (Fig. 1a-b, Supplementary Fig. 1a). These findings are further supported by size exclusion chromatography analyses of the native protein complexes (Fig. 1c, Supplementary Fig. 1b) and suggest mutual exclusivity of complexes containing BRD4-CDK9 and DOT1L. Notably, MLLT1 which has previously been used to co-purify DOT1L and CDK923 was the only 
component shared between the otherwise distinct protein complexes containing BRD4 and DOT1L.

A significant advantage of specific small molecules that target a domain of a protein within a macromolecular complex is that they can engage and negate the functional activity of the protein whilst mostly preserving the native composition of the protein complex. We have previously used this beneficial property to perform chemoproteomics to identify native BRD4 as part of the PTEFb-SEC complex ${ }^{14}$. To extend these data further, we fully characterised the specificity of the DOT1L inhibitor, SGC0946 ${ }^{26}$ (Supplementary Fig. 1c-f, Supplementary Table 1), and functionalised it so that it could be used as an affinity matrix to purify DOT1L and its associated complex members (Supplementary Fig. 2a-b). These interactions were analysed via a chemoproteomic competition binding assay (Fig. 1d, Supplementary Fig. 2c). Together, our chemoproteomic data verify the high specificity of these inhibitors and highlight their utility as molecular probes to interrogate the function of endogenous BRD4 and DOT1L. These data also confirm that DOT1L and BRD4 exist in separate protein complexes albeit with some shared components such as MLLT1 (Fig. 1e).

\section{Targeting BRD4 and DOT1L results in synergy in MLL leukemia}

Having established that BRD4 and DOT1L are components of two distinct protein complexes, we next sought to understand if targeting both of these transcriptional regulators offered any therapeutic advantage. MLL-FP leukemia cells have previously been shown to be the most responsive molecular subtype to either DOT1L or BET-inhibitor therapy, however, the kinetics by which BRD4 and DOT1L inhibition exert their therapeutic effects in MLL-FP leukemia is markedly different ${ }^{14,15,27}$. Therefore, to assess the potential benefit of combination therapy we first established the maximum ineffective dose of I-BET and SGC0946 in various human and mouse leukemia cell lines (Supplementary Fig. 2d-e and data not shown). Using these concentrations, we were able to demonstrate that combination exposure to I-BET and SGC0946 resulted in 
marked synergistic effects that were specific to MLL-FP mouse and human leukemia cell lines (Fig. 2a-d, Supplementary Fig. 2f-h, Supplementary Fig. 3a-d). Importantly, the synergistic activity was also confirmed in clonogenic assays with several primary human AML samples containing various MLL translocations (Fig. 2e).

Whilst in vivo delivery of the pre-clinical BET bromodomain inhibitors has been relatively straightforward ${ }^{14}$, the pharmacokinetic properties of the pre-clinical DOT1L inhibitor, EPZ000477 (closely related to SGC0946), has posed significant challenges to in vivo studies necessitating either ex vivo pretreatment of leukemia cells ${ }^{28}$ or administration via an implantable subcutaneous osmotic mini-pump ${ }^{15}$. We therefore characterized the pharmacokinetic properties of SGC0946 as a single agent or in combination with I-BET therapy in vivo (Supplementary Fig. 3e-f). Using the optimal delivery strategy, we investigated the therapeutic potential of the combination in an aggressive murine model of MLL-AF9 leukemia. Consistent with our in vitro and ex vivo studies, these data show that whilst sub-therapeutic doses of I-BET or SGC0946 offer no survival advantage, combination therapy significantly prolongs survival and reduces disease burden (Fig. 3a-b, Supplementary Fig. 3g).

Previous studies have also used genetic manipulation of BRD4 $4^{27,29}$ or DOT1L 29,30 to demonstrate their individual role as therapeutic targets in MLL-FP leukemia. Using our previously validated inducible shRNAs against BRD431, together with inducible hairpins targeting DOT1L, we replicated these findings. Moreover, in agreement with our data using I-BET and SGC0946, we showed that combined knock-down of BRD4 and DOT1L has a more potent influence on proliferation in vitro (Fig. 3c and Supplementary Fig. 4). To further extend these findings we performed an in vivo competition assay to read out the leukemogenic potential of the RNAi expressing cells. To do this we chose to use a strategy that allows the reliable assessment of the number of RNAi expressing cells at all times during the experiment including the time when the mouse succumbs to the disease. Following the transplant of an equal number of RNAi expressing cells we find at 
multiple time points throughout the experiment, including the time of death, that the number of shRNA expressing cells present in all compartments (peripheral blood, spleen and bone marrow) is lowest in the mice transplanted with cells expressing hairpins against both BRD4 and DOT1L (Fig. 3d-f). Importantly, these data are entirely consistent with our data using small molecules targeting DOT1L and BRD4 and provide compelling pre-clinical evidence that dual targeting of BRD4 and DOT1L provides significantly greater efficacy in MLL-FP leukemia.

\section{BRD4 and DOT1L co-regulate a subset of genes in MLL leukemia}

In order to understand the molecular basis for the observed functional synergy we next sought to investigate the transcription programs regulated by BRD4 and DOT1L. The transcriptional changes following DOT1L or BET inhibition in MV4;11 cells have previously been studied ${ }^{14,15}$ and our findings were highly correlated with these previous studies at identical time points (Supplementary Fig. 5a). In keeping with our proteomic and biochemical data, we find that BRD4 and D0T1L generally control distinct gene expression programs (Fig. 4a). Interestingly, we find that dual targeting of BRD4 and DOT1L results in a more extensive and distinct alteration in gene expression that raised the prospect of a functional interdependency of these two discrete transcriptional complexes (Fig. 4a, Supplementary Fig. 5b). To address this possibility and better understand the molecular events at chromatin, we performed ChIP-Seq analyses following IBET and/or SGC0946 treatment for BRD4 binding, RNA polymerase-II (POL II) occupancy and H3K79me2 density, a histone modification exclusively deposited by D0T1 $\mathrm{L}^{32}$. These data identified three major subsets of genes regulated by the DOT1L and BRD4 containing complexes; those where H3K79me2 is decreased by DOT1L inhibition but BRD4 binding is either absent or unaffected with I-BET treatment, a set of genes where BRD4 binding is decreased by BET inhibition but H3K79me2 is either absent or unaffected within this time frame by SGC0946 treatment and notably a third subset of genes that are co-regulated by both BRD4 and DOT1L (Fig. 4b, Supplementary Table 2). The genes co-regulated by BRD4 and DOT1L inhibition correlated highly with genes that are downregulated 
by combination therapy in our RNA-seq data (Fig. 4c). Importantly, these genes are also highly correlated with genes that have been shown to be directly bound by MLL-FP30 (Fig. 4d) and geneGO analysis further highlighted the fact that these genes are also critically involved in normal and malignant hematopoiesis (Supplementary Fig. 5c).

To further study the effects of BRD4 and/or DOT1L inhibition on transcriptional output in these three subsets of genes, we compared our RNA-Seq and POL II occupancy data with changes in BRD4 displacement and/or a decrease in H3K79me2 (Fig. 4e, Supplementary Fig. 5d). These data demonstrate several important findings. Firstly, BRD4 displacement from chromatin leads to a more significant alteration in transcriptional output than a decrease in H3K79me2 (Fig. 4e-f and Supplementary Fig. 5d-e). As previously reported, we find that H3K79me2 is an excellent marker of actively transcribed genes ${ }^{32}$ (Fig. 5a), however, our data suggests that H3K79me2, by itself, is not required to maintain transcriptional activity at the vast majority of genes (Fig. 4e, Supplementary Fig. 5d-e). Importantly, these data also highlight the fact that dual inhibition of BRD4 and DOT1L results in a dramatic suppression of transcription that is especially notable at the co-regulated genes (Fig. 4e-f, Supplementary Fig. 5d).

\section{H3K79me2 regulates the binding of BRD4 to chromatin}

We next wanted to understand the distinguishing features of the subset of genes that were co-regulated by BRD4 and DOT1L. By assessing POL II occupancy across the coding region of the subset of genes that are regulated by BRD4 and/or D0T1L inhibition, we noted that the co-regulated genes were the most transcriptionally active subset (Fig. 5a). Highly transcribed genes regulated by BRD4 have recently been associated with superenhancers ${ }^{33}$, and our findings are consistent with this observation. Notably however, we can further refine these previous observations by demonstrating that the genes, which are co-regulated by BRD4 and DOT1L in MLL-FP leukemia, are in fact the subset of genes most proximal to a superenhancer (Fig. 5b). Whilst it has clearly been established that 
superenhancers have multiple discriminating features from typical enhancers, including a greater occupancy of transcriptional co-activators such as BRD4 (Supplementary Fig. 6a) and an increased sensitivity to BET bromodomain inhibitors ${ }^{33}$, the regulatory framework governing these intriguing cis-regulatory elements and their associated genes is not fully understood ${ }^{34}$.

H3K79me2 is a histone modification most commonly found throughout the gene body of actively transcribed genes ${ }^{32}$. Interestingly, we found that H3K79me2 was also markedly enriched at transcriptionally active superenhancers compared to typical enhancers (Fig. 5c-d and Supplementary Fig. 6b). Importantly, increased levels of H3K79me2 were detected at superenhancers regardless of whether the superenhancer was an intragenic or intergenic regulatory element (data not shown). Whilst BET inhibition does not lead to a decrease in H3K79me2 levels (Supplementary Fig. 6c), we surprisingly observed that inhibition of DOT1L results in a marked decrease in the binding of BRD4 at superenhancers (Fig. 5e) and their associated genes in human MLL-AF4 and MLL-AF9 driven cells (Supplementary Fig. 6c, e). Importantly, dual inhibition results in a near complete loss of BRD4 and a dramatic suppression of transcription at these sites (Fig. 5e and Supplementary Fig. 6c-d). These intriguing data suggest an unrecognized functional interplay between BRD4 and D0T1L. In further support of this contention, our genome-wide data highlights the specificity of this functional relationship by demonstrating that although IBET results in BRD4 displacement from chromatin regardless of the presence or absence of H3K79me2; DOT1L inhibition only results in BRD4 displacement at genomic regions where H3K79me2 and BRD4 binding are co-located (Fig. 5f).

DOT1L has recently been shown to methylate non-histone proteins ${ }^{35}$ and we therefore wanted to understand whether the ability of DOT1L to regulate BRD4 binding at chromatin was via H3K79 methylation. To address this question, we made use of the fact that SGC0946 has a remarkably long residence time ${ }^{26}$. This feature, coupled with the slow turnover of H3K79me2 (Supplementary Fig. 1d and Supplementary Fig. 7a-b), enabled washout experiments to divorce the 
potential immediate effects of DOT1L inhibition, with unaltered H3K79me2, from the effects mediated by decreased H3K79me2 in the absence of sustained DOT1L inhibition (Supplementary Fig. 7). These data clearly show that D0T1L inhibition, in the absence of decreased H3K79me2, does not alter BRD4 binding (Fig. 5g and Supplementary Fig. 7h). In contrast, when H3K79me2 levels are decreased after SGC0946 washout, we now observe a striking decrease in BRD4 chromatin occupancy at superenhancers and the genes with overlapping BRD4 and H3K79me2 (Fig. 5g and Supplementary Fig. 7h). Taken together, these data demonstrate functional reciprocity between these two transcriptional regulators that is mediated via H3K79me2.

\section{H3K79me2 facilitates histone $\mathrm{H} 4$ acetylation and BRD4 binding}

To understand the molecular mechanism that underpins this functional interdependency, we concentrated our efforts on the genes co-occupied by H3K79me2 and BRD4, whose expression was reduced by DOT1L inhibition. We noticed that the majority of genes whose expression decreased following a reduction in H3K79me2 also had an accompanying loss of BRD4 binding (Supplementary Fig. 8a). To decipher whether this loss of BRD4 binding was required for the transcriptional output of these genes we knocked down BRD4 in human MLL-AF4 and MLL-AF9 driven cells, and assessed transcription at these sites. These data demonstrated that decreased BRD4 levels phenocopies the effects of DOT1L inhibition at these genes (Fig. 6a-b and Supplementary Fig. 8b). Importantly, these data also provide directionality for the sequence of events as the decrease in BRD4 results in a change in gene expression without altering H3K79me2 levels (Fig. 6c) suggesting that BRD4 binding is downstream of H3K79me2 and is directly responsible for modulating gene expression at these loci.

To address the mechanism that links H3K79me2 with chromatin bound BRD4 we first wanted to understand if BRD4 is capable of directly binding H3K79me2. Using histone tail peptides that were unmethylated or mono, di or tri methylated 
at H3K79, we performed immunoprecipitation experiments from nuclear extracts that were then assessed by quantitative mass spectrometry. These data showed that BRD4 does not directly bind H3K79 methylation (Supplementary Fig. 8c). It has previously been established that BRD4 is most avidly associated with chromatin by binding acetylated lysines primarily on the tail of histone H4 and recent data demonstrate that $\mathrm{H} 4 \mathrm{~K} 5 \mathrm{ac}$ is the modification, which is most influential in regulating its avidity for chromatin ${ }^{36,37}$. An established principle in chromatin biology is histone crosstalk whereby the presence or absence of a histone modification may facilitate the deposition or removal of another histone modification ${ }^{38}$. We therefore assessed the possibility that H3K79me2, via histone crosstalk, influenced the deposition of H4K5ac at these loci. Remarkably we found that following DOT1L inhibition, the reduced levels of H3K79me2 led to a concomitant decrease in H4K5ac. These changes were accompanied by decreased BRD4 binding and transcription of the associated genes (Fig. 6d and Supplementary Fig. 8a, d-e). Importantly, this mode of histone crosstalk is also highly specific and confined to regions of the genome where H3K79me2 and H4K5ac overlap with BRD4 binding (Fig. 6e-f and Supplementary Fig. 8a \& f).

To explore the underlying basis for the specific regulation of H4K5ac by H3K79me2, we asked what transcription factor binding sites are most significantly enriched at the areas where H3K79me2 regulates H4K5ac. Notably, the most significant transcription factor binding site enriched in this location was CREB1 (Fig. 7a). Importantly, we confirmed the fact that CREB1 is indeed bound at a number of genes where H3K79me2 influences the deposition of H4K5ac and also found that the binding of CREB1 at these loci is regulated by D0T1L mediated H3K79me2 (Fig. 7b). Furthermore, knock down of CREB1 by two independent and specific shRNAs phenocopied the effects of DOT1L and BRD4 inhibition at these genes (Fig. 7c, Supplementary Fig. 8g). Together these data suggest that H3K79me2 facilitates the DNA binding of transcription factors such as CREB1 at genes that are co-regulated by BRD4 and DOT1L. CREB1 not only plays a role in normal and malignant haematopoiesis ${ }^{39}$ but crucially also 
recruits CREBBP and EP300, which are histone acetyltransferases capable of acetylating histone $\mathrm{H} 4 \mathrm{~K} 5$ and $\mathrm{K} 8^{40,41}$.

Consistent with the possibility that CREB1 recruits EP300 to acetylate H4K5ac, we find that EP300 is bound at the same sites as CREB1 and moreover its recruitment, like that of CREB1, is dependent on DOT1L mediated H3K79me2 (Fig. 7d). In further support of our data, we and others find that RNAi mediated knock down or small molecule inhibition of the catalytic activity of EP300 is highly correlated with the transcriptional effects of inhibiting BRD4 chromatin occupancy (Fig. 7e) ${ }^{41}$. Taken together these data support a model whereby D0T1L methylates histone H3K79 leading to a more permissive chromatin environment for the binding of CREB1 and subsequent recruitment of EP300, which in turn acetylates histone H4K5 and enables the binding of BRD4 to potentiate gene expression at several critical MLL-FP driven oncogenes (Fig. 8).

\section{DISCUSSION}

It is increasingly apparent that a fundamental imbalance in the epigenetic regulation of transcription underpins the molecular pathogenesis of many cancers including acute leukemia. MLL fusion proteins are powerful oncogenes and these leukemias have few other genomic aberrations ${ }^{42}$. The poor prognosis conferred by these leukemias and their relatively simple genetic background have provided the model par excellence for understanding the influence of epigenetic regulators on malignant transcription programs. These studies have yielded several novel epigenetic therapies which are now being investigated across a broad range of malignancies ${ }^{14-17}$. Our clinical experience has highlighted the fact that monotherapy is very unlikely to offer sustained benefit in the management of aggressive malignancies such as MLL-FP leukemia. It has also highlighted the pitfalls of a largely empirical approach to combination therapies especially when these involve the manipulation of context dependent epigenetic regulators ${ }^{8,9}$. Therefore future combination therapies should be based around a comprehensive molecular rationale. 
It has been well established with genetic experiments in sophisticated mouse models that DOT1L has an integral role in MLL-FP leukemia. ${ }^{30,43,44}$ Whilst H3K79me2 has also clearly been demonstrated to be associated with active transcription, what remains uncertain is whether this histone modification is required to maintain the transcriptional activity of these genes. Many histone modifications may simply alter the local chromatin environment or serve to recruit specific factors to chromatin but have little direct effect on transcription. ${ }^{45}$ Our data on H3K79me2 supports the fact that H3K79me2 is an excellent marker of actively transcribed genes, however our results show that DOT1L inhibition leads to a profound decrease in H3K79me2 without a significant change in POL II occupancy or indeed transcription as measured by RNA-Seq suggesting that $\mathrm{H} 3 \mathrm{~K} 79 \mathrm{me} 2$ is not required to directly maintain transcription at the vast majority of these genes. It has recently been demonstrated that H3K79me2 helps maintain an open chromatin state by inhibiting the chromatin localization of the transcriptional repressors SIRT1 and SUV39H128 and consistent with these findings our results suggest that H3K79me2 provides a permissive chromatin state for the recruitment of transcription factors and transcriptional co-activators. Whilst we find that H3K79me2 facilitates the binding of CREB1 and EP300 which acetylates histone H4 for BRD4 localization it is also possible that other transcription factors and/or histone acetyltransferases could be recruited to perform analogous functions. Indeed a recent report has shown that other hematopoietic transcription factors in association with EP300 may also localize BRD4 to potentiate transcription ${ }^{41}$.

Although the effects of BRD4 and DOT1L inhibition has generally been best characterized in the MLL-FP leukemias, there is emerging evidence that therapies against these targets may play a role in other molecular subsets of acute leukemia and indeed other malignancies ${ }^{46-49}$. The differential sensitivity to these therapies in other cancers is presumably because of intrinsic differences in the molecular pathogenesis of these diseases and the fact that there are several 
other protein complexes, which do not contain DOT1L or BRD4, that also nuance and regulate malignant transcription programs initiated by non-MLL-FP oncogenes ${ }^{50,51}$. Nevertheless, whilst our study has focused on MLL-FP leukemia we do not exclude the possibility that that the mechanism we have described here may have broader application in other malignancies and/or normal tissues.

These data also have an immediate clinical relevance, as therapeutic responses to either BET inhibitors ${ }^{52}$ or DOT1L inhibitors ${ }^{53}$ as single agents have now been reported in early clinical trials. As MLL-FP leukemias remain a disease in urgent need for novel therapeutic options, our findings emphasize the fact that targeting of BRD4 and DOT1L in combination negates any functional compensation between these two discrete, yet interdependent, transcription complexes. They provide a unifying mechanism that accounts for the exquisite sensitivity of MLLFP leukemia to DOT1L and BRD4 targeted therapies and specify a molecular framework for future combination epigenetic therapies in the clinical arena.

\section{ACCESSION CODES}

RNA-Seq and ChIP-Seq data have been deposited in the Gene Expression Omnibus database under the accession code GSE71780.

\section{ACKNOWLEDGEMENTS:}

A post-doctoral Fellowship awarded to OG from Leukaemia Foundation Australia partly supported this work. A mid-career Fellowship awarded to EYNL from the Victoria Cancer Agency partly supported this work. A Senior Leukaemia Foundation Australia Fellowship and VESKI Innovation Fellowship currently support MAD. The National Health and Medical Research Council of Australia (1066545 (MAD), 1085015 (MAD), 1106444 (MAD)) and Leukaemia Foundation Australia fund the Dawson lab. This work was also funded in part by the Structural Genomics Consortium a registered charity (no. 1097737) that received funds from AbbVie, Bayer, Boehringer Ingelheim, Genome Canada through the Ontario Genomics Institute (OGI-055), GlaxoSmithKline, Janssen, 
Lilly Canada, the Novartis Research Foundation, the Ontario Ministry of Economic Development and Innovation, Pfizer, Takeda and the Wellcome Trust (092809/Z/10/Z).

\section{AUTHOR CONTRIBUTIONS}

OG, EYNL and MAD designed the research, interpreted data and wrote the manuscript. OG, EYNL, IB, DL, EC, GJ, AW, MW, CYF, SF, DT, KS, LM, C-FW, Y-CC, NG performed experiments and analyzed data. MG, DS, CC, PB, MB, AJB, TK, BJPH, RWJ, GD, SJD, CHA, PG, RKP provided critical reagents and aided in manuscript preparation.

\section{COMPETING FINANCIAL INTEREST}

Isabelle Becher, Dave Lugo, Gerard Joberty, Aoife Ward, Christopher Carpenter, Neil Garton, Gerard Drewes, Paola Grandi, Rab K. Prinjha are employees of GlaxoSmithKline. David Smil, Peter Brown and Cheryl Arrowsmith are employed by the Structural Genomics Consortium, a registered charity (no. 1097737) that received funds from AbbVie, Bayer, Boehringer Ingelheim, Genome Canada through the Ontario Genomics Institute (OGI-055), GlaxoSmithKline, Janssen, Lilly Canada, the Novartis Research Foundation, the Ontario Ministry of Economic Development and Innovation, Pfizer, Takeda and the Wellcome Trust (092809/Z/10/Z). 


\section{References:}

1. Stratton, M.R., Campbell, P.J. \& Futreal, P.A. The cancer genome. Nature 458, 719-24 (2009).

2. Dawson, M.A. \& Kouzarides, T. Cancer epigenetics: from mechanism to therapy. Cell 150, 12-27 (2012).

3. Patel, J.P. et al. Prognostic relevance of integrated genetic profiling in acute myeloid leukemia. $N$ Engl J Med 366, 1079-89 (2012).

4. Cancer Genome Atlas Research, N. Genomic and epigenomic landscapes of adult de novo acute myeloid leukemia. $N$ Engl J Med 368, 2059-74 (2013).

5. Cai, S.F., Chen, C.W. \& Armstrong, S.A. Drugging Chromatin in Cancer: Recent Advances and Novel Approaches. Mol Cell 60, 561-70 (2015).

6. Dawson, M.A., Kouzarides, T. \& Huntly, B.J. Targeting epigenetic readers in cancer. $N$ Engl J Med 367, 647-57 (2012).

7. Rathert, P. et al. Transcriptional plasticity promotes primary and acquired resistance to BET inhibition. Nature 525, 543-7 (2015).

8. Prebet, T. et al. Prolonged administration of azacitidine with or without entinostat for myelodysplastic syndrome and acute myeloid leukemia with myelodysplasia-related changes: results of the US Leukemia Intergroup trial E1905. J Clin Oncol 32, 1242-8 (2014).

9. Issa, J.P. et al. Results of phase 2 randomized study of low-dose decitabine with or without valproic acid in patients with myelodysplastic syndrome and acute myelogenous leukemia. Cancer 121, 556-61 (2015).

10. Yu, B.D., Hess, J.L., Horning, S.E., Brown, G.A. \& Korsmeyer, S.J. Altered Hox expression and segmental identity in Mll-mutant mice. Nature 378, 505-8 (1995).

11. McMahon, K.A. et al. Mll has a critical role in fetal and adult hematopoietic stem cell self-renewal. Cell Stem Cell 1, 338-45 (2007).

12. Jude, C.D. et al. Unique and independent roles for MLL in adult hematopoietic stem cells and progenitors. Cell Stem Cell 1, 324-37 (2007).

13. Neff, T. \& Armstrong, S.A. Recent progress toward epigenetic therapies: the example of mixed lineage leukemia. Blood 121, 4847-53 (2013).

14. Dawson, M.A. et al. Inhibition of BET recruitment to chromatin as an effective treatment for MLL-fusion leukaemia. Nature 478, 529-33 (2011).

15. Daigle, S.R. et al. Selective killing of mixed lineage leukemia cells by a potent small-molecule DOT1L inhibitor. Cancer Cell 20, 53-65 (2011).

16. Grembecka, J. et al. Menin-MLL inhibitors reverse oncogenic activity of MLL fusion proteins in leukemia. Nat Chem Biol 8, 277-84 (2012).

17. Harris, W.J. et al. The histone demethylase KDM1A sustains the oncogenic potential of MLL-AF9 leukemia stem cells. Cancer Cell 21, 473-87 (2012).

18. Mohan, M., Lin, C., Guest, E. \& Shilatifard, A. Licensed to elongate: a molecular mechanism for MLL-based leukaemogenesis. Nat Rev Cancer 10, 721-8 (2010).

19. Biswas, D. et al. Function of leukemogenic mixed lineage leukemia 1 (MLL) fusion proteins through distinct partner protein complexes. Proc Natl Acad Sci U S A 108, 15751-6 (2011). 
20. Bitoun, E., Oliver, P.L. \& Davies, K.E. The mixed-lineage leukemia fusion partner AF4 stimulates RNA polymerase II transcriptional elongation and mediates coordinated chromatin remodeling. Hum Mol Genet 16, 92-106 (2007).

21. Lin, C. et al. AFF4, a component of the ELL/P-TEFb elongation complex and a shared subunit of MLL chimeras, can link transcription elongation to leukemia. Mol Cell 37, 429-37 (2010).

22. Mohan, M. et al. Linking H3K79 trimethylation to Wnt signaling through a novel Dot1-containing complex (DotCom). Genes Dev 24, 574-89 (2010).

23. Mueller, D. et al. A role for the MLL fusion partner ENL in transcriptional elongation and chromatin modification. Blood 110, 4445-54 (2007).

24. Mueller, D. et al. Misguided transcriptional elongation causes mixed lineage leukemia. PLoS Biol 7, e1000249 (2009).

25. Yokoyama, A., Lin, M., Naresh, A., Kitabayashi, I. \& Cleary, M.L. A higherorder complex containing AF4 and ENL family proteins with P-TEFb facilitates oncogenic and physiologic MLL-dependent transcription. Cancer Cell 17, 198-212 (2010).

26. $\mathrm{Yu}, \mathrm{W}$. et al. Catalytic site remodelling of the DOT1L methyltransferase by selective inhibitors. Nat Commun 3, 1288 (2012).

27. Zuber, J. et al. RNAi screen identifies Brd4 as a therapeutic target in acute myeloid leukaemia. Nature 478, 524-8 (2011).

28. Chen, C.W. et al. DOT1L inhibits SIRT1-mediated epigenetic silencing to maintain leukemic gene expression in MLL-rearranged leukemia. Nat Med 21, 335-43 (2015).

29. Shi, J. et al. Discovery of cancer drug targets by CRISPR-Cas9 screening of protein domains. Nat Biotechnol 33, 661-7 (2015).

30. Bernt, K.M. et al. MLL-rearranged leukemia is dependent on aberrant H3K79 methylation by DOT1L. Cancer Cell 20, 66-78 (2011).

31. Fong, C.Y. et al. BET inhibitor resistance emerges from leukaemia stem cells. Nature 525, 538-42 (2015).

32. Nguyen, A.T. \& Zhang, Y. The diverse functions of Dot1 and H3K79 methylation. Genes Dev 25, 1345-58 (2011).

33. Loven, J. et al. Selective inhibition of tumor oncogenes by disruption of super-enhancers. Cell 153, 320-34 (2013).

34. Pott, S. \& Lieb, J.D. What are super-enhancers? Nat Genet 47, 8-12 (2015).

35. Yang, L. et al. IncRNA-dependent mechanisms of androgen-receptorregulated gene activation programs. Nature 500, 598-602 (2013).

36. LeRoy, G. et al. Proteogenomic characterization and mapping of nucleosomes decoded by Brd and HP1 proteins. Genome Biol 13, R68 (2012).

37. Zhao, R., Nakamura, T., Fu, Y., Lazar, Z. \& Spector, D.L. Gene bookmarking accelerates the kinetics of post-mitotic transcriptional re-activation. Nat Cell Biol 13, 1295-304 (2011).

38. Lee, J.S., Smith, E. \& Shilatifard, A. The language of histone crosstalk. Cell 142, 682-5 (2010).

39. Shankar, D.B. et al. The role of CREB as a proto-oncogene in hematopoiesis and in acute myeloid leukemia. Cancer Cell 7, 351-62 (2005). 
40. Henry, R.A., Kuo, Y.M. \& Andrews, A.J. Differences in specificity and selectivity between CBP and p300 acetylation of histone $\mathrm{H} 3$ and H3/H4. Biochemistry 52, 5746-59 (2013).

41. Roe, J.S., Mercan, F., Rivera, K., Pappin, D.J. \& Vakoc, C.R. BET Bromodomain Inhibition Suppresses the Function of Hematopoietic Transcription Factors in Acute Myeloid Leukemia. Mol Cell 58, 1028-39 (2015).

42. Lavallee, V.P. et al. The transcriptomic landscape and directed chemical interrogation of MLL-rearranged acute myeloid leukemias. Nat Genet 47, 1030-7 (2015).

43. Jo, S.Y., Granowicz, E.M., Maillard, I., Thomas, D. \& Hess, J.L. Requirement for Dot1l in murine postnatal hematopoiesis and leukemogenesis by MLL translocation. Blood 117, 4759-68 (2011).

44. Nguyen, A.T., Taranova, O., He, J. \& Zhang, Y. DOT1L, the H3K79 methyltransferase, is required for MLL-AF9-mediated leukemogenesis. Blood 117, 6912-22 (2011).

45. Bannister, A.J. \& Kouzarides, T. Regulation of chromatin by histone modifications. Cell Res 21, 381-95 (2011).

46. Dawson, M.A. et al. Recurrent mutations, including NPM1c, activate a BRD4-dependent core transcriptional program in acute myeloid leukemia. Leukemia 28, 311-20 (2014).

47. McLean, C.M., Karemaker, I.D. \& van Leeuwen, F. The emerging roles of DOT1L in leukemia and normal development. Leukemia 28, 2131-8 (2014).

48. Shi, J. \& Vakoc, C.R. The mechanisms behind the therapeutic activity of BET bromodomain inhibition. Mol Cell 54, 728-36 (2014).

49. Sarkaria, S.M., Christopher, M.J., Klco, J.M. \& Ley, T.J. Primary acute myeloid leukemia cells with IDH1 or IDH2 mutations respond to a DOT1L inhibitor in vitro. Leukemia 28, 2403-6 (2014).

50. Jonkers, I. \& Lis, J.T. Getting up to speed with transcription elongation by RNA polymerase II. Nat Rev Mol Cell Biol 16, 167-77 (2015).

51. Smith, E. \& Shilatifard, A. Transcriptional elongation checkpoint control in development and disease. Genes Dev 27, 1079-88 (2013).

52. Herait, P.E. et al. BET-bromodomain inhibitor OTX015 shows clinically meaningful activity at nontoxic doses: interim results of an ongoing phase I trial in hematologic malignancies. Cancer Res 74, CT231 (2014).

53. Stein, E.M. et al. The D0T1L Inhibitor EPZ-5676: Safety and Activity in Relapsed/Refractory Patients with MLL-Rearranged Leukemia. Blood 124(2014). 


\section{FIGURE LEGENDS:}

\section{Figure 1: BRD4 and DOT1L are in separate protein complexes}

(a) Heat map summary of quantitative (iTRAQ) proteomic analysis of immunoprecipitation experiments (IP). Endogenous BRD4 or DOT1L were immunoprecipitated from HL-60 nuclear extracts, enrichment of bait proteins as well as selected known interactors of bait proteins is shown (see also Table S1A). (b) Coimmunoprecipitation of endogenous BRD4, and MLLT1 in MOLM-13 cells. IgG was used as a control. Immunoblot (IB) analysis with antibodies against DOT1L. (See also Supplementary Data Set 1). (c) Size exclusion chromatography performed on HEL nuclear extracts followed by immunoblot analysis of BRD4, DOT1L, and CDK9. (d) Chemoproteomic competition binding assay. Mass spectrometry analysis of eluates from HL-60 nuclear extracts incubated with the SGC0946 matrix in the presence of DMSO or increasing concentrations of SGC0946. (e) Scatterplot of chemoproteomic enrichment of proteins captured via a SGC0946 matrix or a I-BET matrix and competition with the respective free compound.

Figure 2: Inhibition of BRD4 and DOT1L leads to synergistic efficacy against MLL-FP leukemia cell lines and primary patient cells

(a) Proliferation assay performed with human MV4;11 cells treated with DMSO, I-BET, SGC0946 or both. Mean, error bars, s.d. (n = 3 cell culture replicates), representative graph from experiments done on 3 separate occasions. (b) Proliferation assay of mouse MLL-AF9 leukemia cells. Low dose and high dose respectively were defined in Supplementary Figure 2. For I-BET, LD $=100 \mathrm{nM}$ and HD $=500 n M$. For SGC0946, LD $=1 \mu \mathrm{M}$ and HD $=5 \mu \mathrm{M}$. Mean, error bars, s.d. ( $n=3$ cell culture replicates), representative graph from experiments done on 3 separate occasions. (c) Proliferation assay with K562 driven by BCR-ABL and (d) KG-1 cells. Mean, error bars, s.d. ( $\mathrm{n}=3$ cell culture replicates), representative graph from experiments done on 3 separate occasions. (e) Clonogenic assays. primary human MLL-FP AML cells were plated in the presence of vehicle (DMSO), I-BET (LD), SGC0946 (LD) or combination of I-BET (LD) and SGC0946 
(LD). Mean ( $\mathrm{n}=2$ cell culture replicates).

Figure 3: Inhibition of BRD4 and DOT1L by small molecule and shRNA leads to synergistic efficacy against MLL-FP leukemia in vivo

(a) Kaplan-Meier curve of vehicle and drug treated C57BL/6 mice transplanted with $2 \times 10^{6}$ MLL-AF9 leukemic cells. Black arrows represent treatment with IBET at a low dose of $10 \mathrm{mg} / \mathrm{kg} /$ day via IP injections and shaded orange region represents treatment with SGC0946 at $6 \mathrm{mg} / \mathrm{kg} / \mathrm{h}$ via continuous osmotic pump delivery, $n=5$ mice per group. $P$ values are calculated using log-rank test. (b) Spleen weights of the mice from the various treatment cohorts. $n=5$ mice per group. The upper limit, center and lower limit of boxplots denote the upper quartile, median and lower quartile of the data, respectively. Whiskers extend to maximum and minimum values. P-value by two-tailed Student's $t$ test. (c) Competition assay of inducible shScramble with shBRD4 and/or shD0T1L in mouse MLL-AF9 cells treated with doxycycline for 8 days. dsRED positive cells were normalized to day 1 post doxycycline exposure. Mean, error bars, s.d. (n=3 cell culture replicates), representative graph from experiments done on 2 separate occasions. (d-f) Percentage of shRNA+/dsRED+ cells post transplant of leukemic cells. The upper limit, center and lower limit of boxplots denote the upper quartile, median and lower quartile of the data, respectively. Whiskers extend to maximum and minimum values. Percentage of shRNA+/dsRED+ cells in the (d) peripheral blood at day 18, (e) spleen at endpoint and (f) bone marrow at endpoint. $n=5$ mice per group.

Figure 4: A subset of genes is responsive to both BRD4 and DOT1L inhibition.

(a) RNA-Seq Principal component analysis of MV4;11 cells treated with I-BET, SGC0946, or a combination of I-BET and SGC0946, and vehicle control in experiments done on 2 separate occasions. (b) ChIP-Seq for H3K79me2 and BRD4 after treatment with I-BET or SGC0946. Top, venn diagram of the number of genes in each of the three categories of BRD4 and DOT1L regulated genes. Bottom, ChIP-Seq profiles of an exemplar gene from each category. (c) Gene set enrichment analysis (GSEA) of the down-regulated genes following combination 
treatment of MV4;11 cells with the co-regulated subset of genes. (d) GSEA of the genes down-regulated by combination treatment with previously published MLL-AF9 fusion target genes ${ }^{30}$. (e) Log-fold changes in BRD4 binding (BRD4 LFC) and H3K79me2 levels (H3K79me2 LFC) in genes from ChIP-Seq are plotted against the change in POL II (POL II LFC) binding following I-BET, SGC0946 or combination treatment. The numbers of genes in each quadrant are shown. (f) Waterfall plot demonstrating the change in POL II (POL II LFC) binding throughout the gene body of the co-regulated genes, ranked by increasing POL II LFC, following I-BET, SGC0946 or combination treatment.

\section{Figure 5: DOT1L regulates BRD4 binding at superenhancers via H3K79me2}

(a) Median POL II coverage (RPKM) across the four subsets of BRD4 and DOT1L regulated genes in vehicle treated cells from ChIP-Seq. Regions $5 \mathrm{~kb}$ upstream of the TSS (transcription start site) to 5kb downstream of the TES (transcriptional end site) are in $250 \mathrm{bp}$ bins. The gene body is scaled to bins consisting of $1 \%$ of gene length. (b) Distribution of distance to the nearest superenhancer for the four subsets of BRD4 and DOT1L regulated genes. (c) The median coverage of H3K79me2 (RPKM) from ChIP-Seq across superenhancers and typical enhancers with $5 \mathrm{~kb}$ upstream and downstream in untreated cells. (d) 4sU RNA-Seq coverage in enhancers with and without H3K79me2. The upper limit, center and lower limit of boxplots denote the upper quartile, median and lower quartile of the data, respectively. Whiskers extend to $1.5 \times$ interquartile range above and below the upper and lower quartiles, respectively. (e) Median coverage (RPKM) of BRD4 binding from ChIP-Seq across superenhancers and typical enhancers with $5 \mathrm{~kb}$ upstream and downstream following I-BET, SGC0946 or combination treatment. (f) BRD4 binding in genomic regions where only BRD4 is present compared to regions where BRD4 and H3K79me2 occur together. The upper limit, center and lower limit of boxplots denote the upper quartile, median and lower quartile of the data, respectively. Whiskers extend to $1.5 \times$ interquartile range above and below the upper and lower quartiles, respectively. (g) Median BRD4 coverage (RPKM) at superenhancers with H3K79me2, following 8 hours SGC0946 treatment or 72 hours post SGC0946 washout. 


\section{Figure 6: H3K79me2 regulates BRD4 binding via H4 acetylation}

(a) GSEA of the genes down-regulated by SGC0946 with genes down-regulated by BRD4 shRNA knock-down in MV4;11 cells. (b) shRNA mediated knockdown of BRD4 in MOLM-13 cells. Bar graphs represent the relative mRNA levels . Mean, error bars, s.d. ( $\mathrm{n}=3$ experiments done on separate occasions) ${ }^{*} \mathrm{P}<0.05,{ }^{* *} \mathrm{P}<$ 0.01 by two-tailed student's $t$ test. (c) qPCR analysis of H3K79me2 ChIP using primers against CDK6, MTHFD2 and ATF4. Mean, error bars, s.d. $(\mathrm{n}=3$ experiments done on separate occasions). (d) ChIP-Seq profile of CDK6 with SGC0946 treatment in MV4;11 cells. (e) Log-fold changes in the levels of H4K5ac upon SGC0946 treatment in genomic regions where only BRD4 is present compared to regions where BRD4 and H3K79me2 occur together. The upper limit, center and lower limit of boxplots denote the upper quartile, median and lower quartile of the data, respectively. Whiskers extend to $1.5 \times$ interquartile range above and below the upper and lower quartiles, respectively. (f) Metagene profiles of BRD4 and H3K79me2 normalised read counts centred on regions with H4K5 acetylation.

\section{Figure 7: H3K79me2 regulates BRD4 binding via EP300 mediated H4 acetylation}

(a) MEME Motif analysis. Left, Enrichment of the CREB1 motif in positive genomic regions with both BRD4 and H3K79me2 where BRD4 binding and H4K5ac levels are lost following SGC0946 treatment. Negative regions represent those where H4K5ac levels are unaltered. Right, Sequence logo of the CREB1 motif. (b) qPCR of CREB1 ChIP using primers against CDK6, MTHFD2 and ATF4 and Negative control region (neg) in MV4;11 cells. Mean, error bars, s.d. ( $n=3$ experiments done on separate occasions). (c) qRT-PCR of CREB1 knockdown by two independent shRNAs using primers against CDK6, MTHFD2 and ATF4 in MV4;11 cells. Bar graphs represent the relative mRNA levels. Mean, error bars, s.d. ( $n=3$ experiments done on separate occasions). (d) qPCR analysis of EP300 ChIP using primers against CDK6, MTHFD2, ATF4 and B2M. Mean, error bars, s.d. ( $n=3$ experiments done on separate occasions). (e) GSEA of genes down- 
regulated by shRNA-mediated BRD4 knock down in mouse MLL-AF9 cells with genes previously found to be down-regulated by Ep300 knock down or small molecule inhibition in the same leukemia model ${ }^{31}$.

Figure 8: Schematic model for the functional interdependency of DOT1L and BRD4 in MLL-Leukemia

H3K79 is methylated by DOT1L, which results in a more open and permissive chromatin environment for the binding of transcription factors such as CREB1. This in turn results in the recruitment of EP300, which catalyses the acetylation H4K5. BRD4 and its associated protein complex subsequently binds to H4K5ac to facilitate the transcription of target genes. 


\section{ONLINE METHODS}

\section{Generation of immortalised primary murine haematopoietic progenitor cell lines}

Initial generation of an immortalised parental cell line was achieved through magnetic bead selection (Miltenyi Biotec) of c-kit positive cells, obtained from whole bone marrow of male and female C57BL/6 mice, and subsequent retroviral transduction with a MSCV-MLL-AF9-IRES-YFP construct.

\section{Cell culture}

Primary murine haematopoietic progenitors and derived cell lines were grown in RPMI-1640 supplemented with murine IL-3 (10 ng/mL), 20\% fetal calf serum, penicillin (100 units $/ \mathrm{mL})$, streptomycin $(100 \mu \mathrm{g} / \mathrm{mL})$, amphotericin $B$ (250ng/mL) and gentamycin $(50 \mu \mathrm{g} / \mathrm{mL})$. Primary human leukemia cells were grown in the presence of IL3 (10 ng/mL), IL6 (10 ng/mL) and SCF (50 ng/mL). Cells were incubated at $37^{\circ} \mathrm{C}$ and $5 \% \mathrm{CO}_{2}$. MV4-11, MOLM-13, KG-1, NB-4, HL-60 and SKM-1 cells were grown in RPMI-1640 supplemented with $20 \%$ fetal calf serum, glutamax, penicillin (100units $/ \mathrm{mL})$, and streptomycin $(100 \mu \mathrm{g} / \mathrm{mL})$. Trypan Blue staining was performed for all cell counting. 4\% Trypan Blue was added to a sample of cell culture media at 1:1 ratio, and cell count calculated by BioRad automated cell counter. All cell lines were authenticated by short tandem repeat (STR) profiling using the AmpFISTR ${ }^{\circledR}$ dentifier ${ }^{\circledR}$ kit, applied biosystems, and tested for mycoplasma contamination every two months.

\section{Cell proliferation assays}

Cells were seeded at $5 \times 10^{4}$ cells $/ \mathrm{mL}$ in duplicates and treated with DMSO, IBET, SGC0946 or both and counted daily for 4-6 days using a BioRad automated cell counter.

\section{Clonogenic assays in methylcellulose}


Clonogenic potential was assessed through colony growth of human patient samples plated in cytokine-supplemented methylcellulose (Methocult M3434, Stemcell Technologies). Human patient MLL samples were plated in duplicate at a cell dose of $2 \times 10^{2}$ per plate in the presence of vehicle (0.1\% DMSO) or drug (750nM I-BET151, $1 \mu$ M SGC0946 or both). Cells were incubated at $37^{\circ} \mathrm{C}$ and $5 \%$ $\mathrm{CO}_{2}$ for 7 to 10 days at which time colonies were counted.

\section{Flow cytometric analyses}

Cell apoptosis was assessed using APC conjugated Annexin V (550475, BD Biosciences) and propidium iodide (PI) (P4864, Sigma-Aldrich) staining according to manufacturer's instructions. Induction of apoptosis in human leukemia cell lines was determined following treatment with either vehicle (DMSO) or 500nM I-BET151 for 24 to 48 hours in liquid culture. For cell cycle analysis, cells were fixed overnight at $-20^{\circ} \mathrm{C}$ in $70 \%$ EtOH/PBS. Prior to flow cytometry analysis, cells were incubated at $37^{\circ} \mathrm{C}$ for 30 mins in PI solution (0.02mg/mL PI, $0.05 \% \mathrm{v} / \mathrm{v}$ Triton-X in PBS, supplemented with DNase-free RNAse A (19101, Qiagen)). Flow cytometry analyses were performed on a LSRFortessa X-20 flow cytometer (BD Biosciences) and all data analysed with FlowJo software (Tree Star). Cell sorting was performed on a FACSAria Fusion flow sorter (BD Biosciences).

\section{Size exclusion chromatography}

Cell lysates were separated on a superose- 6 column and fractions collected were analysed by western blot for the presence of proteins of interest.

\section{Chemoproteomics}

HL-60 nuclear extracts have been prepared, and immunoprecipitations of BRD4 and DOT1L performed, as previously described ${ }^{14}$. Briefly, antibodies (antiBRD414; anti-DOT1L, Novus Biologicals \#NB100-40845) and a control rabbit IgG (Sigma \#15006) were covalently coupled to 100ul AminoLink resin (Thermo 
Fisher Scientific \#20501). The immobilized antibodies were incubated with HL60 nuclear extracts for 3 hours, rotating at $4^{\circ} \mathrm{C}$. Beads were washed and protein was recovered for LC-MS/MS analysis.

Affinity matrices were generated and affinity profiling assays were performed with HL-60 nuclear extracts as described previously ${ }^{14}$. Beads were derivatized with $20 \mu \mathrm{M}$ GSK2691981, an analogue of GSK1210151, or 1 mM GSK3338813A (see Supplementary Notes), an analogue of SGC0946.

LC-MS/MS analysis: Sample preparation, labeling with isobaric mass tags, peptide fractionation, and mass spectrometric analyses were performed essentially as described ${ }^{54,55}$. All experiments (except BRD4 IP) were performed using the TMT $^{\text {тм }}$ (Thermo-Fisher Scientific) multiplex option to allow comparison of up to 10 conditions in one analysis ${ }^{56}$.

Peptide and protein identification: Mascot 2.4 (Matrix Science, Boston, MA) was used for protein identification by using a 10 parts per million mass tolerance for peptide precursors and $20 \mathrm{mD}$ (HCD) mass tolerance for fragment ions. Carbamidomethylation of cysteine residues and TMT modification of lysine residues were set as fixed modifications and methionine oxidation, and $\mathrm{N}$ terminal acetylation of proteins and TMT modification of peptide $\mathrm{N}$-termini were set as variable modifications. The search database consisted of a customized version of the International Protein Index protein sequence database combined with a decoy version of this database created by using a script supplied by Matrix Science. Unless stated otherwise, we accepted protein identifications as follows: (i) For single-spectrum to sequence assignments, we required this assignment to be the best match and a minimum Mascot score of 31 and a $10 \times$ difference of this assignment over the next best assignment. Based on these criteria, the decoy search results indicated $<1 \%$ false discovery rate (FDR). (ii) For multiple spectrum to sequence assignments and using the same parameters, the decoy search results indicate $<0.1 \%$ FDR. All identified proteins were quantified; FDR for quantified proteins was $<1 \%$.

Peptide and protein quantification: Reporter ion intensities were read from raw data and multiplied with ion accumulation times (the unit is milliseconds) so as to yield a measure proportional to the number of ions; this measure is referred 
to as ion area ${ }^{57}$. Spectra matching to peptides were filtered according to the following criteria: mascot ion score $>15$, signal-to-background of the precursor ion $>4$, and signal-to-interference $>0.5^{58}$. Fold-changes were corrected for isotope purity as described and adjusted for interference caused by co-eluting nearly isobaric peaks as estimated by the signal-to-interference measure 59 . Protein quantification was derived from individual spectra matching to distinct peptides by using a sum-based bootstrap algorithm; 95\% confidence intervals were calculated for all protein fold-changes that were quantified with more than three spectra ${ }^{57}$.

For visualization only proteins with $\geq 2$ peptides and $\geq 3$ spectra were considered in heatmap and scatter plot representation (Figure 1a and e).

\section{Co-immunoprecipitation}

$4 \times 10^{7}$ MV411 or MOLM-13 cells were harvested. Cells were washed in ice cold PBS and centrifuged at $400 \mathrm{~g}$ for 5 minutes, $4 \mathrm{C}(\times 2)$ and lysed in $600 \mu \mathrm{L}$ of Modified HEPES Lysis Buffer, (appendix II) with (1x) Protease inhibitor cocktail solution (Roche) for 2 minutes. Cell debris was precipitated by centrifugation at maximum speed $(15,000 \mathrm{rpm})$ for $10 \mathrm{~min}$. A $100 \mu \mathrm{L}$ sample was collected for Western Blot input control, before being diluted with $500 \mu \mathrm{L}$ of $50 \mathrm{M}$ Tris Buffer. $1 \mathrm{~mL}$ of supernatant was aliquoted and split between two eppendorf tubes. $40 \mu \mathrm{L}$ Magnetic Dynabeads®;M-280 Strepdavidin (Invitrogen) were washed in HEPES lysis wash buffer $(3 x)$ and then incubated with lysates for 3 hours at 4 degrees on spinning rotator (SB3, Stuart). After incubation magnetic beads were precipitated using a Dyna Mag 2 magnet (Life technologies). Samples were washed in modified HEPES wash lysis buffer $(2 \times)$ utilizing Dyna Mag 2 for bead separation; final pellet was re-suspended in $50 \mathrm{ul}$ of wash lysis buffer. $10 \mu \mathrm{L}$ of SDS sample buffer was added to bead bound protein 'sample' solution, and $20 \mu \mathrm{L}$ to input sample, and incubated at 95 degrees for 5 minutes.

\section{Immunoblotting}


Whole cell lysates were mixed with Laemmli buffer, separated via SDS-PAGE, transferred to PVDF membranes (Millipore). Membranes were then sequentially incubated with primary antibodies (see antibodies) and secondary antibodies conjugated with horseradish peroxidase (Invitrogen). Membranes were then incubated with ECL (GE Healthcare) and proteins detected by exposure to x-ray film.

\section{RNAi studies}

shRNAs were cloned into a tet-on inducible vector backbone, TtRMPVIR (27995, addgene). For competitive proliferation assays, transduced cells were sorted for shRNA-containing $\left(\right.$ Venus $^{+} / \mathrm{YFP}^{+}$or $\mathrm{BFP}^{+} / \mathrm{YFP}^{+}$) and non shRNA-containing $\left(\mathrm{YFP}^{+}\right.$only) populations and recombined at a 1:1 ratio. Following this, cells were cultured with $1 \mathrm{mg} / \mathrm{mL}$ doxycycline to induce shRNA expression. The proportion of shRNA-expressing (dsRED ${ }^{+} /$Venus $\left.^{+} / \mathrm{YFP}^{+}\right)$cells were determined by flow cytometric analysis and followed over time. Knockdown efficiency of shRNAexpressing and non shRNA-containing cells was assessed following 72 hours of doxycycline exposure by qRT-PCR and immunoblotting. Sequence of shRNA in Supplementary Notes.

\section{qRT-PCR}

mRNA was prepared using the Qiagnen RNeasy kit and cDNA synthesis was performed using SuperScript VILO kit (Life Technologies) as per manufacturers' instructions. Quantitative PCR analysis was undertaken on an Applied Biosystems StepOnePlus System with SYBR green reagents. For analysis of murine cell line samples, expression levels were determined using the $\Delta \mathrm{CT}$ method and normalised to beta-2-microglobulin and/or GAPDH. Assessment of expression changes associated with I-BET151 treatment occurred at 6 hours following treatment with 500nM I-BET151 and/or SGC0946 for 72-96hrs. Sequence of primers in Supplementary Notes. 


\section{SGC0946 Washout}

Cells were cultured for 6, 8 or 10hrs with SGC0946, after which the compound was washed out and cells replaced with fresh media. Cells were then cultured up to $72 \mathrm{hrs}$ and harvested. Short-term treatment was performed on the same batch of cells just prior to harvesting.

\section{Antibodies}

The following antibodies were used in ChIP assays: anti-BRD4 (E2A7X, cell signalling), anti-H3K27ac (ab4729, abcam), Anti-H3K79me2 (ab3594, abcam), anti-RNA POL II (CTD4H8, Millipore). Immunobloting assays: anti-BRD4 (A301985A, Bethyl Labs and ab128874, abcam) anti-HSP60 (sc-13966, Santa Cruz Biotechnology), anti-H3K79me2 (ab3594, abcam), anti-MYC (9402S, Cell signalling technologies), anti-CDK9 (A303-492A, Bethyl Labs), anti-LE01 (A300174A, Bethyl Labs), anti-MLLT1 (A302-267A, Bethyl Labs), anti-D0T1L (ab72454, abcam), Anti-IgG rabbit (Santa Cruz Biotechnology), anti-P300 (ab14984, abcam), anti-H4K5ac (ab51997, abcam), anti-CREB1 (48H2, cell signalling). Validation is provided on the manufacturer's website.

\section{Pharmacokinetic optimization of SGC0946 delivery}

The systemic exposure of SGC0946 was determined in male CD1 mice ( $\mathrm{n}=3$ per dose route) following oral (PO), subcutaneous (SC) or intraperitoneal (IP) dosing at a target dose of $3 \mathrm{mg} / \mathrm{kg}$ ( $5 \mathrm{~mL} / \mathrm{kg}$ ) for all 3 routes. SGC0946 was suspended in 1\% methylcellulose 400 (aq) or dissolved in 10\% (w/v) Kleptose HPB for the PO and SC/IP dose groups respectively. The systemic exposure of SGC0946 post insertion of a surgically implanted minipump was determined in male C57BLK mice (approximately $25 \mathrm{~g}, \mathrm{n}=3$ ) at a target dose of $6 \mathrm{mg} / \mathrm{kg} / \mathrm{h}(1 \mu \mathrm{L} / \mathrm{h})$. SGC0946 was dissolved in 10\% (w/v) Kleptose HPB to achieve a final concentration of 150 $\mathrm{mg} / \mathrm{mL}$. Serial blood samples (15 or $20 \mu \mathrm{L}$ ) were taken via the tail vein over the time-course. Blood samples were diluted with an equal volume of water and stored at $\mathrm{ca} .-20^{\circ} \mathrm{C}$ prior to analysis by LC-MS/MS. 
Blood samples were analysed for SGC0946 using an analytical method based on protein precipitation and LC-MS/MS employing positive-ion electrospray ionisation using an Phenomenex Kinetix C18 LC column and a Sciex API 4000 MS. Pharmacokinetic data analysis was performed using non-compartmental analysis in WinNonLin Phoenix v6.2 software.

\section{Syngeneic murine models of leukemia}

Secondary syngeneic transplantation studies were performed with intravenous injection of $2 \times 10^{6}$ cells obtained from bone marrow or spleen into C57BL/6 male mice, all mice were 6-10weeks old at time of sublethal irradiation at a dose of 3Gy. Treatment with vehicle, I-BET151, SGC0946 or both began at day 8. IBET was administered via intra-peritoneal injection at $10 \mathrm{mg} / \mathrm{kg} /$ day on days 8 , 9, 11,14 and 16. SGC0946 was administered via osmotic mini-pumps surgically implanted, and were filled with $150 \mathrm{mg} / \mathrm{ml}$ to deliver a dose of $6 \mathrm{mg} / \mathrm{kg} / \mathrm{h}$. Pumps were implanted at day 8 and left for the duration of the experiment. All mice were kept in a pathogen free animal facility, inspected daily and sacrificed upon signs of distress and disease. All studies were conducted in accordance with the GSK Policy on the Care, Welfare and Treatment of Laboratory Animals and were reviewed by the Institutional Animal Care and Use Committee either at GSK or were conducted under institutional animal ethics review board in Australia and authorised by the Animal Experimentation Ethics Committee (AEEC), Peter MacCallum Cancer Centre. All mice were randomised prior to commencement of experiment. Animal technicians were blinded to experimental setup and outcome.

\section{Inducible shRNA in vivo competition assay}

Secondary MLL-AF9 cells were transduced with shScramble, shDOT1L, shBRD4 or both shDOT1l and shBRD4. Cells were sorted for BFP+, VENUS+ or BFP+/VENUS+. Doxocycline was then added to cells in vitro for $48 \mathrm{hrs}$ and DSRED+ cells were sorted and immediately transplanted into NSG mice. Mice 
were kept on DOX for the duration of the experiment. Peripheral blood was taken every 9 days.

\section{Combination treatment for RNA-Seq and ChIP-Seq}

MV4;11 cells were seeded at $1-2 \times 10^{5}$ per $\mathrm{ml}$ and treated with DMSO or SGC0946 $(5 \mu \mathrm{M})$ for $72 \mathrm{hrs}$, with I-BET $(500 \mathrm{nM})$ treatment commencing $6 \mathrm{hrs}$ prior to harvesting alone or in combination.

\section{RNA-Seq}

RNA for RNA-Seq was prepared using the Qiagen RNeasy kit. RNA concentration was quantified with the NanoDrop spectrophotometer (Thermo Scientific). The integrity was assessed with the 2100 bioanalyzer using the RNA 6000 kit (Agilent Technologies). Libraries were prepared using the TruSeq RNA library preparation kit (Illumina). Libraries were quantified by qPCR, normalised and pooled to $2 \mathrm{nM}$ before sequencing with single-end $50 \mathrm{bp}$ reads on an Illumina HiSeq2500. All RNA-seq experiments were done in duplicate.

\section{RNA-Seq analysis}

Reads were aligned to the human genome (G1k V37) using tophat2 and bowtie2 and reads were assigned to genes using htseq-count ${ }^{42}$. Differential expression was calculated using edgeR ${ }^{60}$. Genes with a false discovery rate corrected for multiple testing using the method of Benjamini and Hochberg61 below 0.05 and a fold-change greater than 1.5 were considered significantly differentially expressed.

Principal component analysis was performed on the variance stabilizing transformed read counts ${ }^{62}$. Gene set testing using ROAST ${ }^{63}$ was performed on voom-transformed RNA-Seq data. 
Diseases significantly associated with the genes co-regulated by I-BET and SGC0946 were identified by MetaCore (Thomson Reuters) enrichment analysis.

\section{Chromatin Immunoprecipitation (ChIP)}

Cells were cross-linked with $1 \%$ formaldehyde for 15 minutes at room temperature and cross-linking stopped by the addition of $0.125 \mathrm{M}$ glycine. Cells were then lysed in 1\% SDS, $10 \mathrm{mM}$ EDTA, 50mM Tris- $\mathrm{HCl}$ pH8.0 and protease inhibitors. Lysates were sonicated in a Covaris ultrasonicator to achieve a mean DNA fragment size of 500bp. Immunoprecipitation (see antibodies) was performed for a minimum of 12 hours at $4^{\circ} \mathrm{C}$ in modified RIPA buffer (1\% Triton $\mathrm{X}, \quad 0.1 \%$ deoxycholate, $90 \mathrm{mM} \mathrm{NaCl}, 10 \mathrm{mM}$ Tris- $\mathrm{HCl}$ pH8.0 and protease inhibitors). An equal volume of protein $A$ and $G$ magnetic beads (Life Technologies) were used to bind the antibody and associated chromatin. Reverse crosslinking of DNA was followed by DNA purification using QIAquick PCR purification kits (Qiagen). Immunoprecipitated DNA was analysed by sequencing (see below) or qPCR on an Applied Biosystems StepOnePlus System with SYBR green reagents. All ChIP-Seq experiments were performed once. Sequence of primers in Supplementary Notes.

\section{ChIP-Seq analysis}

Reads were aligned to the human genome (G1k V37) using bowtie264. Peak calling was performed using MACS2 with default parameters ${ }^{65}$. Genome browser images of ChIP-Seq data was generated by converting the bam files from bowtie2 to TDF files using igvtools and viewing in IGV66. ChIP-Seq coverage across selected genomic regions was calculated using BEDtools ${ }^{67}$.

\section{Defining subsets of I-BET and SGC0946 regulated genes}

ChIP coverage for POL II, BRD4 and H3K79me2 was calculated using BEDTools ${ }^{67}$ in all expressed ensemble genes including $1 \mathrm{~kb}$ promoter region and normalized for read depth and gene length. Genes were filtered to only include those with a 
minimum PolII ChIP reads per kilobase per million reads (RPKM) of of 1 (corresponding to top $50 \%$ of expressed genes). Genes with a ChIP-Seq fold change comparing inhibitor-treated with DMSO-treated cells of at least -1.3 (corresponding to the top $10 \%$ of most regulated genes) were considered regulated. Gene set enrichment analysis was performed with GSEA ${ }^{68}$ or ROAST ${ }^{63}$.

\section{Gene coverage profiles}

Genes were divided into 100 segments between the TSS and TES, and $5 \mathrm{~kb}$ upstream and downstream of the gene were divided into 20 intervals of $250 \mathrm{bp}$ using a python script. ChIP-Seq coverage was counted for each of the intervals for each gene using BEDTools ${ }^{67}$. The median number of reads normalized for 1 million reads in $1 \mathrm{~kb}$ region was calculated across all genes, and plotted for different sets of regulated genes using ggplot $2{ }^{69}$ in the R statistical programming language ${ }^{70}$.

\section{Define superenhancer}

Peaks identified by MACS2 in BRD4 ChIP in DMSO sample were intersected with K27ac ChIP to identify the enhancers with both BRD4 and K27ac. Peaks within $12.5 \mathrm{~kb}$ were merged, promoter regions within $2.5 \mathrm{~kb}$ of TSS were removed. Enhancer regions with BRD4 coverage in DMSO sample with reads per million (RPM) of $>3500$ were defined as superenhancers. A total of 332 superenhancers were identified in MV4;11 cells.

\section{Enhancer coverage profiles}

The median size of superenhancers is approximately 25 times greater than the size of typical enhancers. Therefore superenhancers were divided into 100 intervals, typical enhancers were divided into 4 intervals and 5kb upstream and downstream of the gene were divided into 20 intervals of $250 \mathrm{bp}$ using a python script. Coverage of ChIP reads per kilobase per million reads (RPKM) was 
calculated over superenhancers and typical enhancers using BEDTools ${ }^{67}$ and the median was plotted for different treatments.

\section{ChIP-Seq profile plots}

Profiles of ChIP-Seq reads in the $5 \mathrm{~kb}$ around the H4K5ac MACS peaks were generated with Genomic Tools ${ }^{71}$.

\section{Regulated gene numbers}

Levels of H3K79me2, BRD4 and H4K5ac were computed in ChIP-Seq peaks within expressed genes using BEDTools and summed for each gene with $\mathrm{R}^{70}$. Genes with a decrease of ChIP-Seq reads of 1.3 fold were considered to be significant.

\section{4sU labelling of newly transcribed RNA}

4sU labelling was performed to isolate newly transcribed RNA ${ }^{72}$. MV4;11 cells treated with DMSO, 500nM I-BET for 6 hours and/or 5uM SGC0946 for 3 days, and labelled for 60 minutes with $200 \mu \mathrm{M} 4 \mathrm{sU}$ at the end of inhibitor treatment. Total RNA was isolated from with TRIzol@ reagent. The RNA was denatured by heating to $65^{\circ} \mathrm{C}$ for 10 minutes, then placed on ice. The labelled RNA was biotinylated with $2 \mu \mathrm{g}$ EZ-link HPDP-biotin (Thermo Fisher) per $\mu \mathrm{g}$ of RNA in biotinylation buffer for 1.5 hours at RT. The biotinylated RNA was extracted with an equal volume of chloroform twice, precipitated with ethanol, then resuspended in water. The biotinylated RNA was purified with $25 \mu \mathrm{L}$ of Dynabeads M-280 streptavidin (Thermo Fisher), washed four times with washing buffer (5mM Tris- $\mathrm{HCl}$ pH7.5, $0.5 \mathrm{mM}$ EDTA and $1 \mathrm{M} \mathrm{NaCl})$. The biotinylated RNA was eluded from the beads with 100mM DTT and purified with RNAeasy MinElute cleanup (Qiagen) kit. RNA concentration was quantified with the NanoDrop spectrophotometer (Thermo Fisher). The integrity was assessed with the 2100 bioanalyzer using the RNA 6000 kit (Agilent Technologies). cDNA was synthesised from the recovered newly synthesised RNA with random 
hexamers. Libraries were prepared using the TruSeq RNA library preparation kit (Illumina). Libraries were quantified by qPCR, normalized and pooled to $2 \mathrm{nM}$ before sequencing with single-end 50 bp reads on an Illumina HiSeq2500. All $4 \mathrm{sU}$ experiments were done in duplicate.

\section{Motif analysis}

Motif analysis was performed with CentriMo ${ }^{73}$ on genomic regions with both BRD4 and H3K79me2 where BRD4 binding and H4K5ac levels are lost following SGC0946 treatment, compared with regions where H4K5ac levels are unaltered.

\section{Patient material}

Peripheral blood or bone marrow containing $>80 \%$ blasts was obtained from patients following consent and under full ethical approval by the Peter MacCallum Cancer Centre human research ethics committee. 


\section{Supplementary References:}

54. Kruse, U. et al. Chemoproteomics-based kinome profiling and target deconvolution of clinical multi-kinase inhibitors in primary chronic lymphocytic leukemia cells. Leukemia 25, 89-100 (2011).

55. Savitski, M.M. et al. Tracking cancer drugs in living cells by thermal profiling of the proteome. Science 346, 1255784 (2014).

56. Werner, T. et al. High-resolution enabled TMT 8-plexing. Anal Chem 84, 7188-94 (2012).

57. Savitski, M.M. et al. Delayed fragmentation and optimized isolation width settings for improvement of protein identification and accuracy of isobaric mass tag quantification on Orbitrap-type mass spectrometers. Anal Chem 83, 8959-67 (2011).

58. Savitski, M.M. et al. Targeted data acquisition for improved reproducibility and robustness of proteomic mass spectrometry assays. $J$ Am Soc Mass Spectrom 21, 1668-79 (2010).

59. Savitski, M.M. et al. Measuring and managing ratio compression for accurate iTRAQ/TMT quantification. J Proteome Res 12, 3586-98 (2013).

60. Robinson, M.D., McCarthy, D.J. \& Smyth, G.K. edgeR: a Bioconductor package for differential expression analysis of digital gene expression data. Bioinformatics 26, 139-40 (2010).

61. Benjamini, Y.H., Yosef. Controlling the False Discovery Rate: A Practical and Powerful Approach to Multiple Testing. J. Roy. Statist. Soc. Ser. B 57, 289-300 (1995).

62. Anders, S. \& Huber, W. Differential expression analysis for sequence count data. Genome Biol 11, R106 (2010).

63. $\mathrm{Wu}, \mathrm{D}$. et al. ROAST: rotation gene set tests for complex microarray experiments. Bioinformatics 26, 2176-82 (2010).

64. Langmead, B. \& Salzberg, S.L. Fast gapped-read alignment with Bowtie 2. Nat Methods 9, 357-9 (2012).

65. Zhang, Y. et al. Model-based analysis of ChIP-Seq (MACS). Genome Biol 9, R137 (2008).

66. Robinson, J.T. et al. Integrative genomics viewer. Nat Biotechnol 29, 24-6 (2011).

67. Quinlan, A.R. \& Hall, I.M. BEDTools: a flexible suite of utilities for comparing genomic features. Bioinformatics 26, 841-2 (2010).

68. Subramanian, A. et al. Gene set enrichment analysis: a knowledge-based approach for interpreting genome-wide expression profiles. Proc Natl Acad Sci U S A 102, 15545-50 (2005).

69. Wickham, H. ggplot2: elegant graphics for data analysis, (Springer New York, 2009).

70. Team, R.C. R: A Language and Environment for Statistical Computing. (ed. Computing, R.F.f.S.) (Vienna, Austria, 2015).

71. Tsirigos, A., Haiminen, N., Bilal, E. \& Utro, F. GenomicTools: a computational platform for developing high-throughput analytics in genomics. Bioinformatics 28, 282-3 (2012).

72. Radle, B. et al. Metabolic labeling of newly transcribed RNA for high resolution gene expression profiling of RNA synthesis, processing and decay in cell culture. J Vis Exp (2013). 
73. Bailey, T.L. \& Machanick, P. Inferring direct DNA binding from ChIP-seq. Nucleic Acids Res 40, e128 (2012). 
Figure 1

a IP with antibody for: BRD4 DOT1L

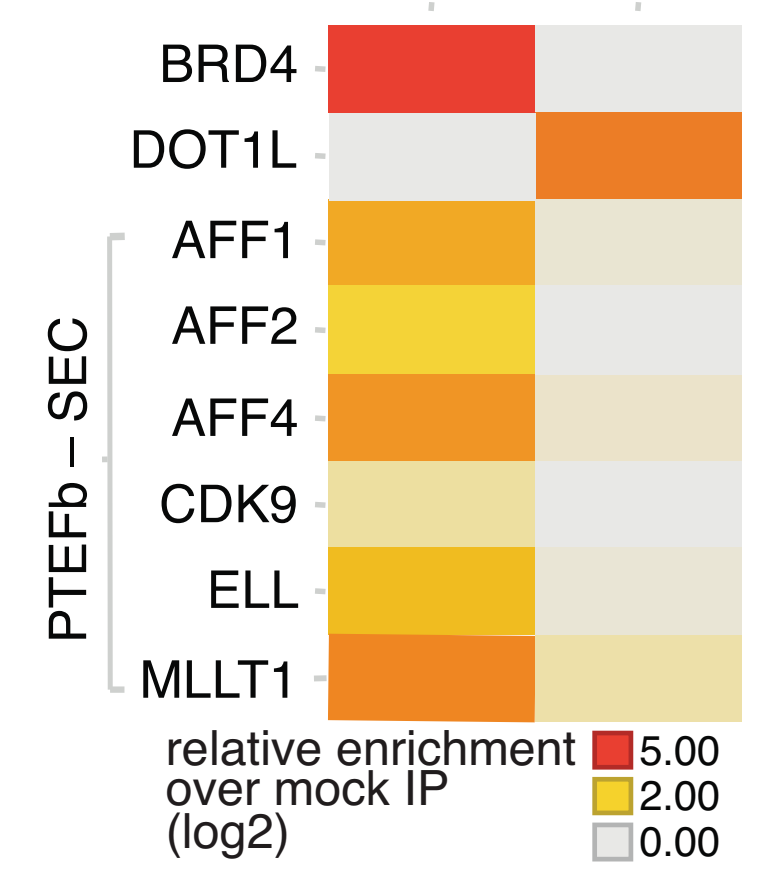

d

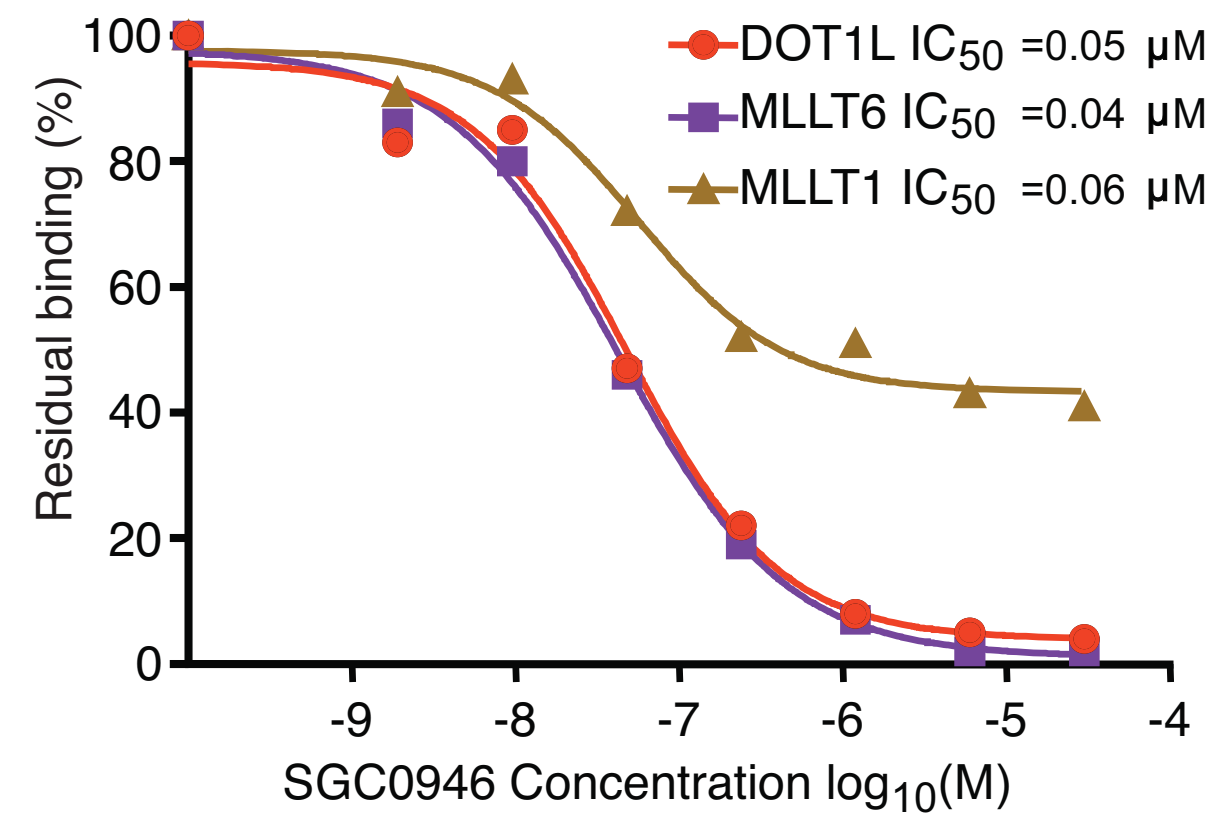

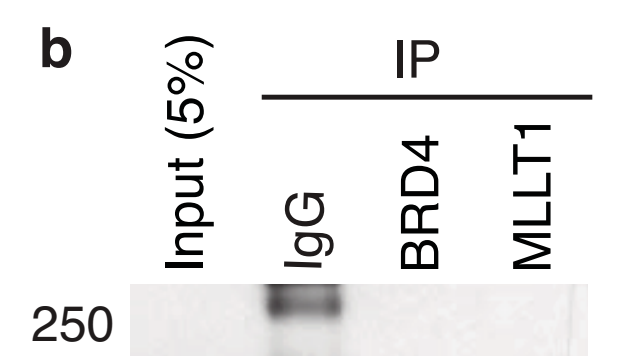

C

MLL (WT)

\section{$670 \mathrm{kDa}$}

Fractions

10111213

$\mathrm{I}$

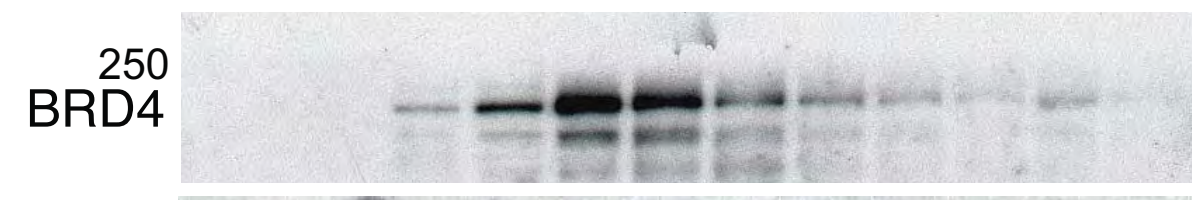

55
CDK9

95

250
DOT1L

\section{IB: DOT1L}

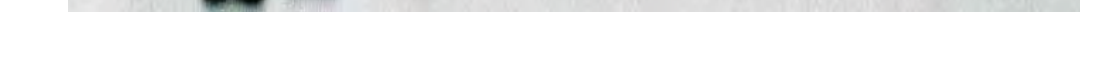

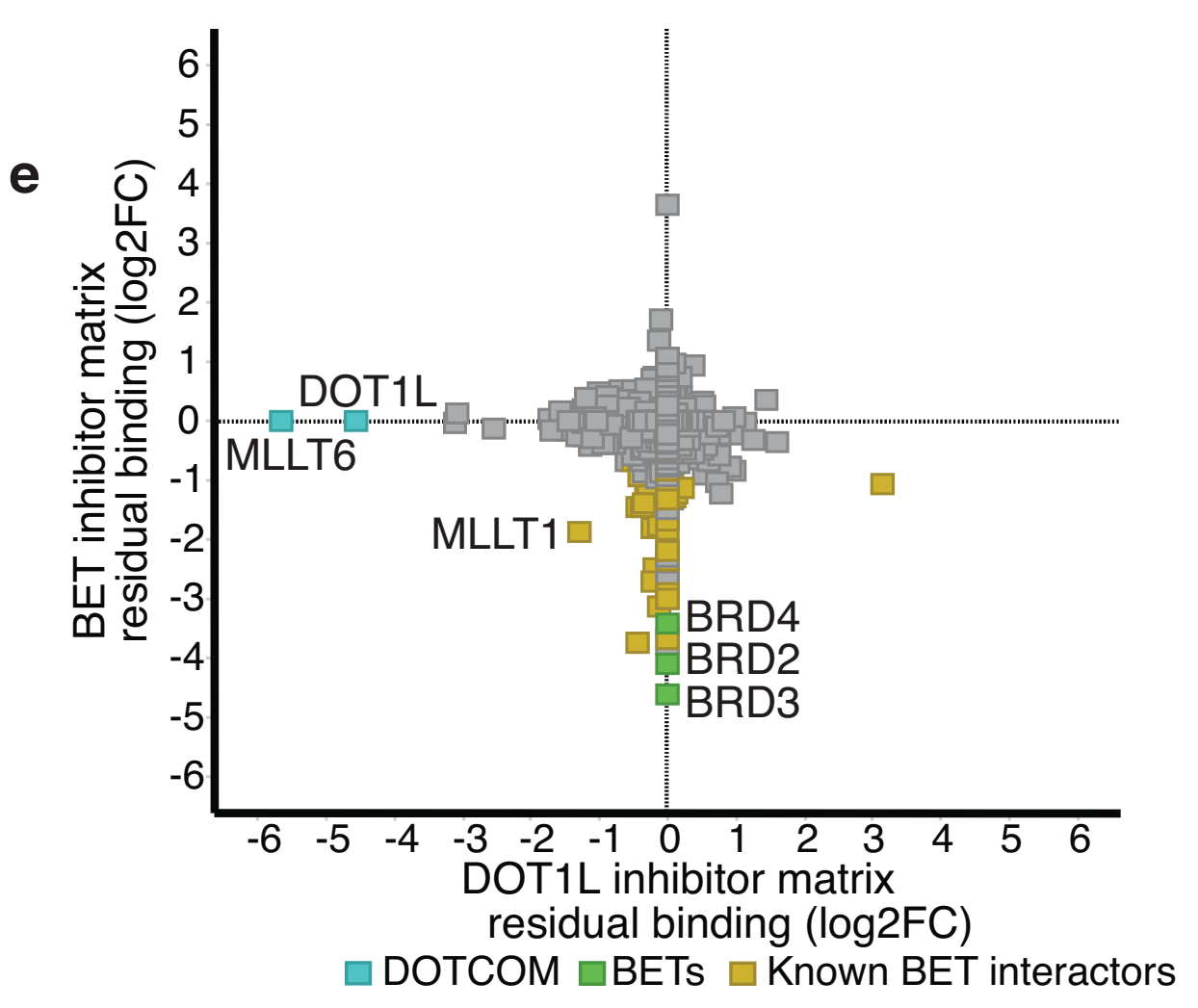


a

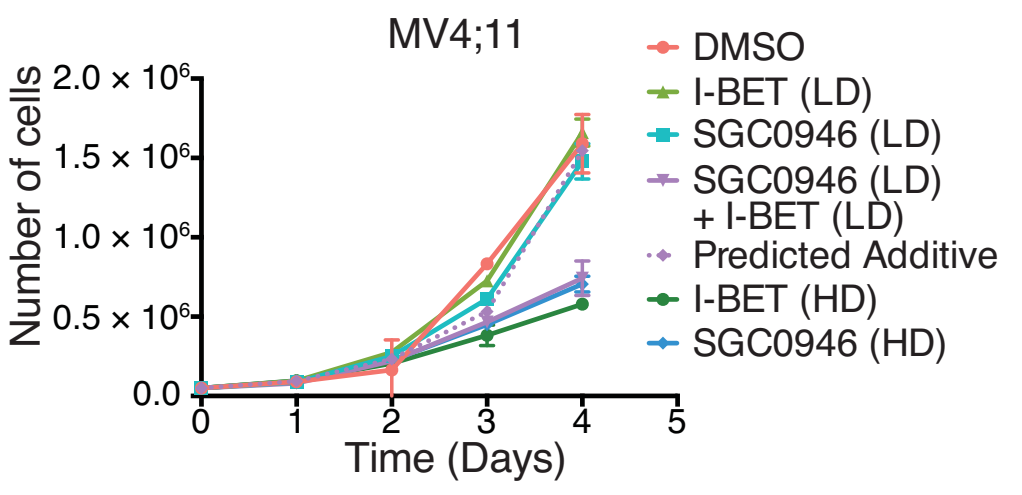

C

K562
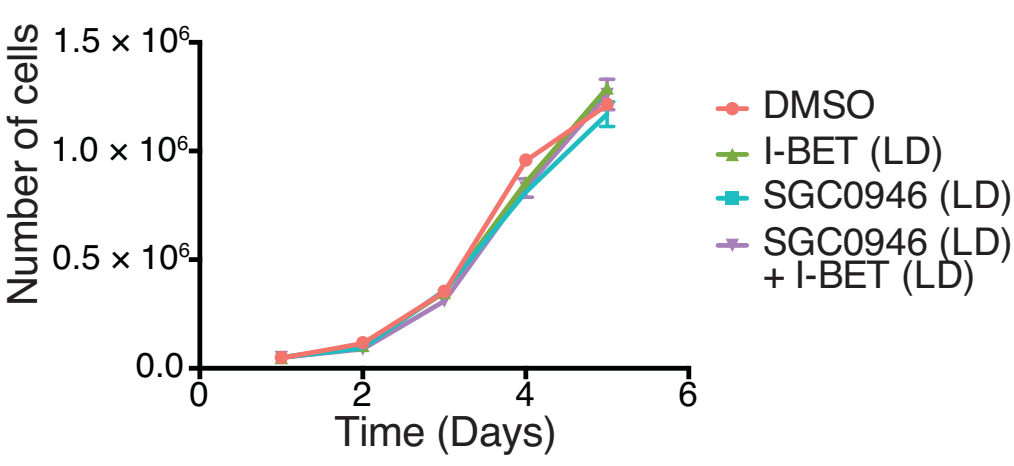

b

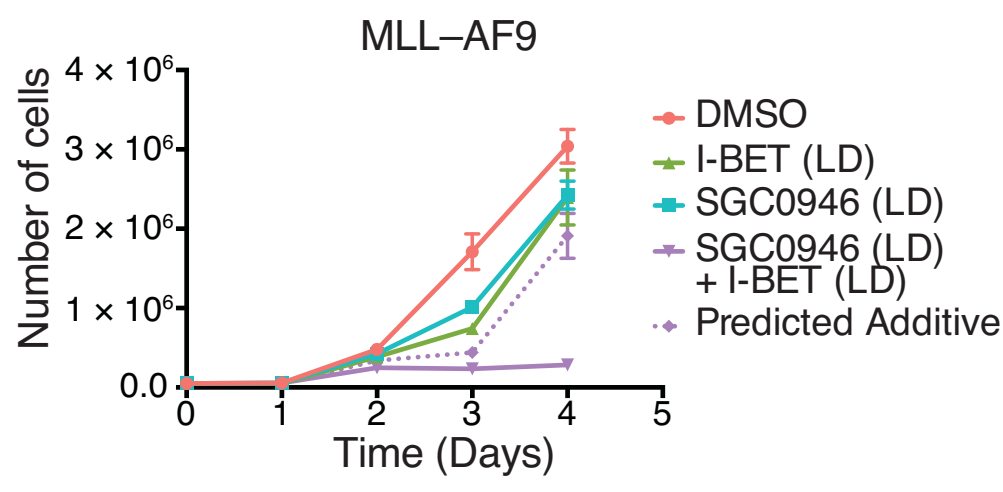

d
- DMSO

- I-BET (LD)

- SGC0946 (LD)

- SGC0946 (LD) + I-BET (LD)
MLL-SEPT6

2
MLL-AF10

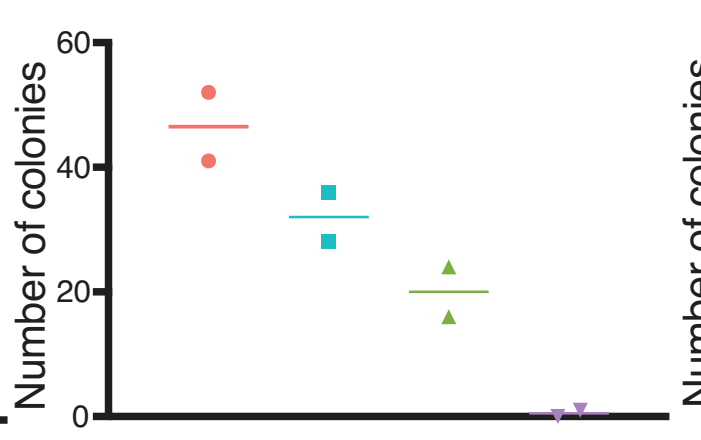

MLL-AF9

MLL-AF9

MLL-ELL

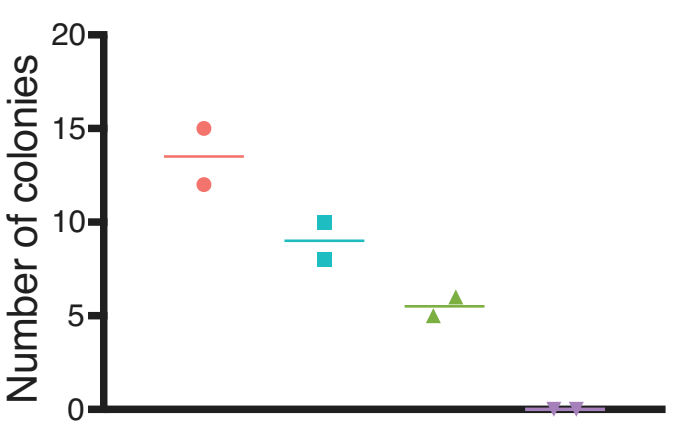

DMSO

SGC0946 (LD)

I-BET (LD)

SGC0946 (LD) + I-BET (LD) 
Figure 3

a

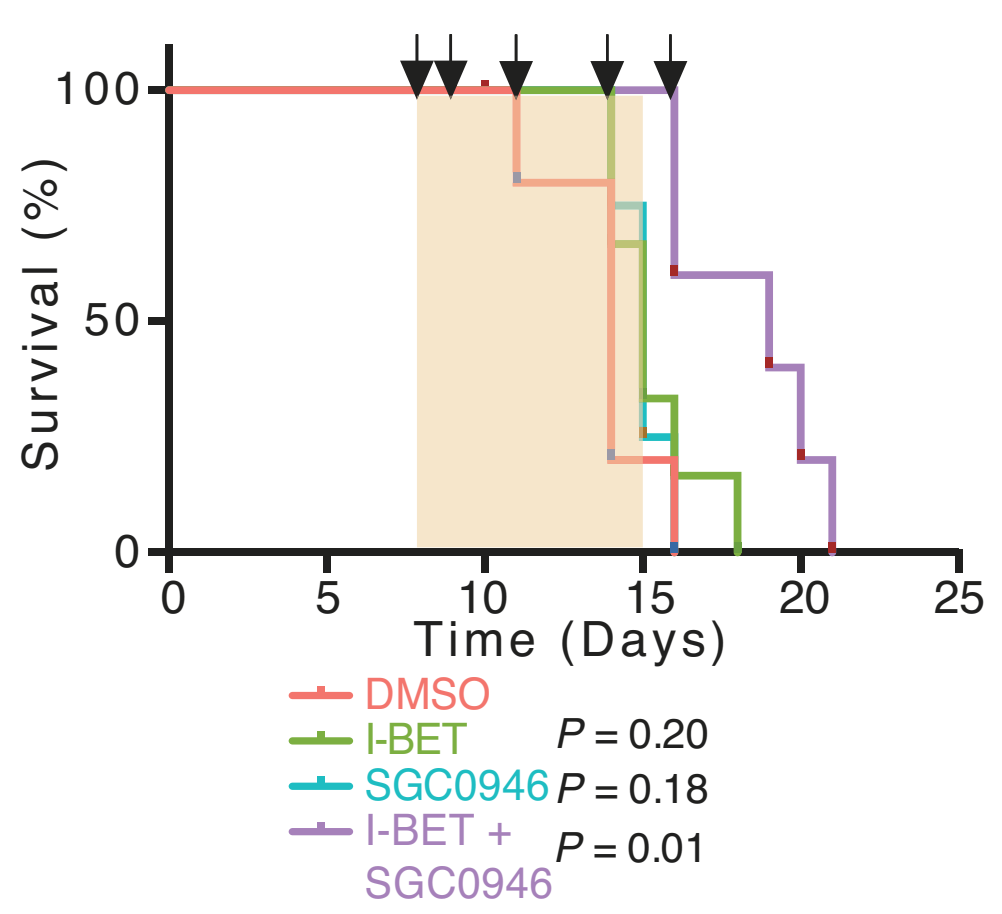

d

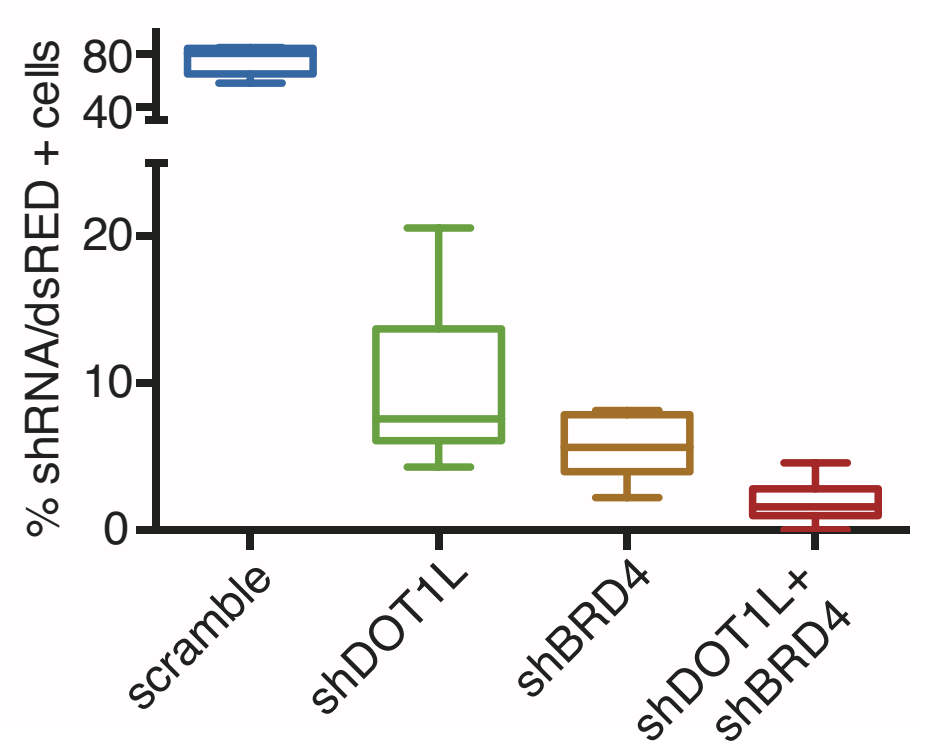

b

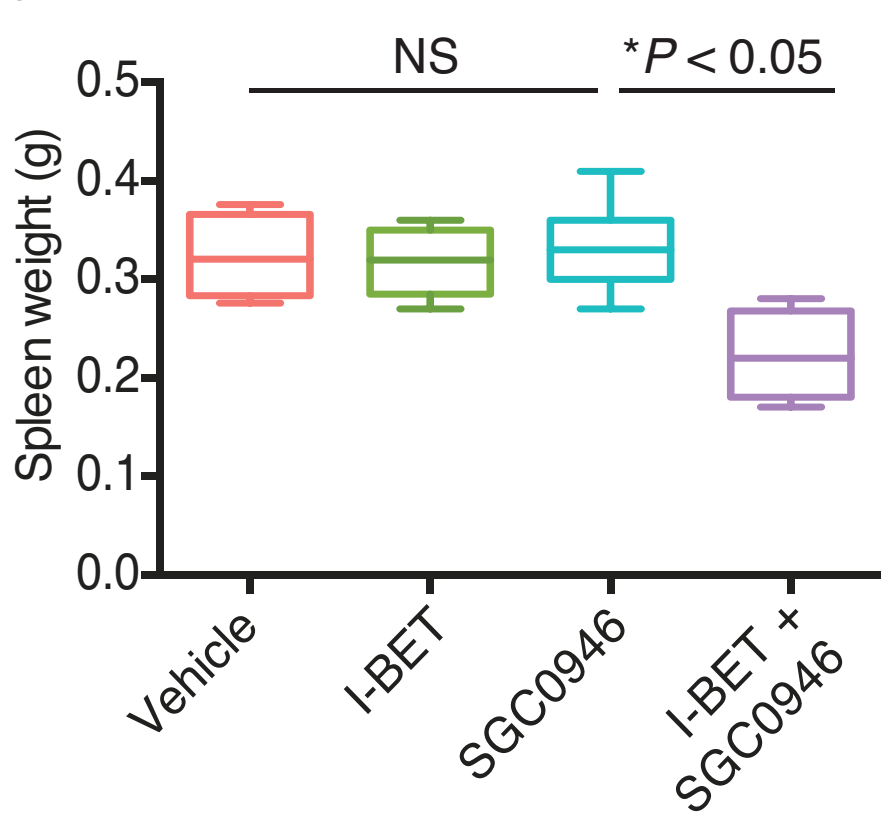

e

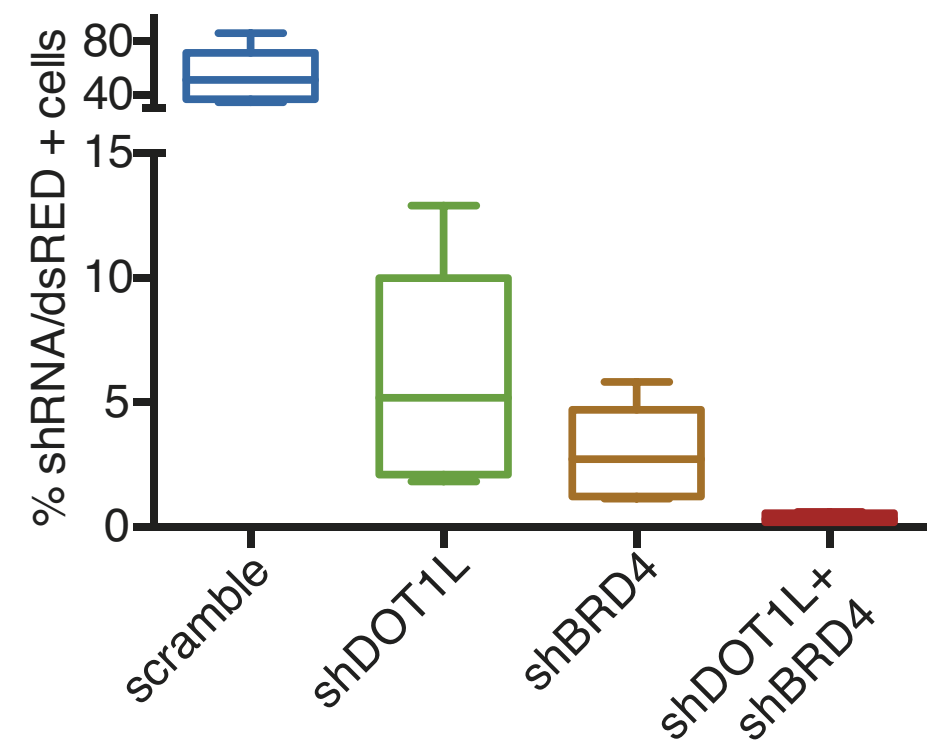

C

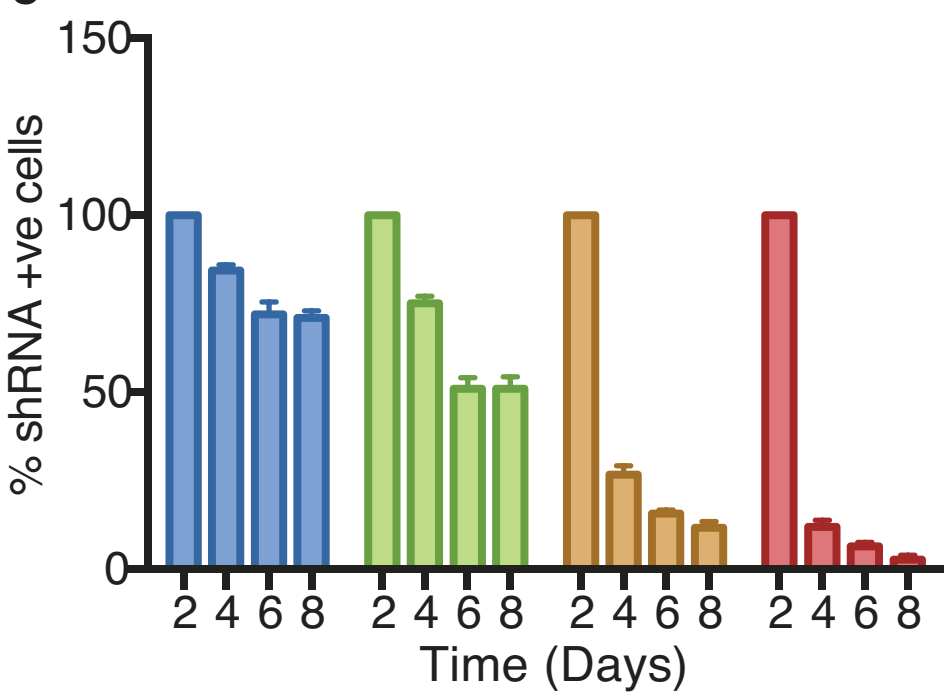

$\square$ shScramble $\square$ shBRD4 \#498

$\square$ shDOT1L \#7 shBRD4/shDOT1L

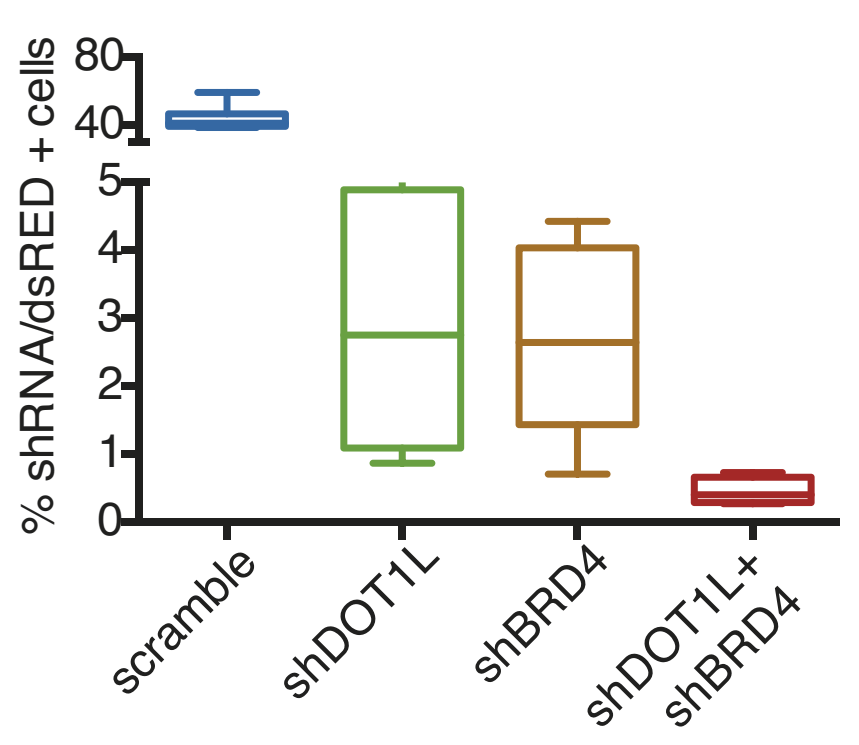


Figure 4

\section{a}

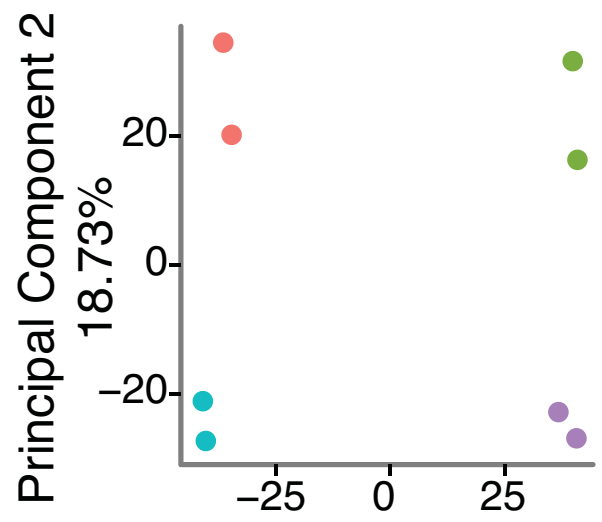

Principal Component 1 $42.46 \%$

C

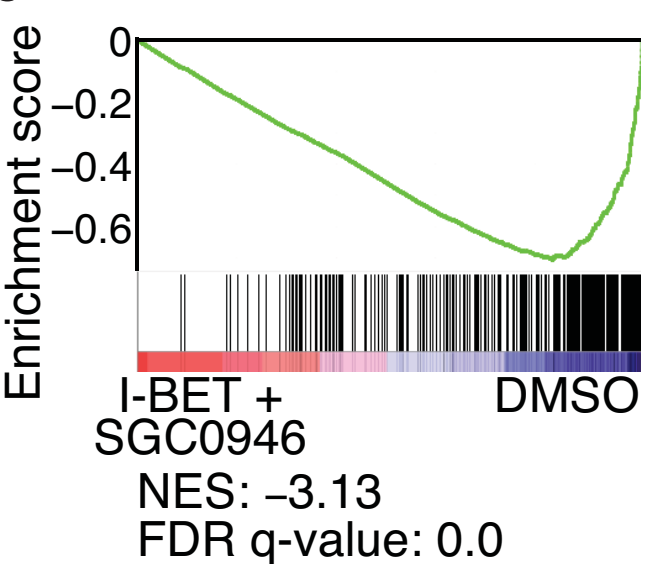

d

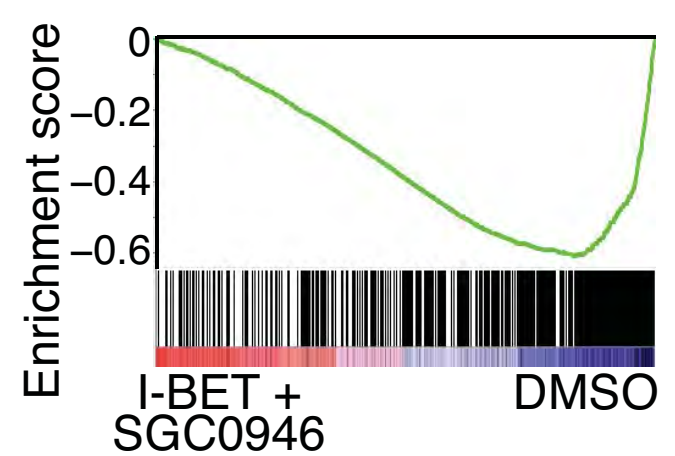

NES: -2.86

FDR q-value: 0.0 b

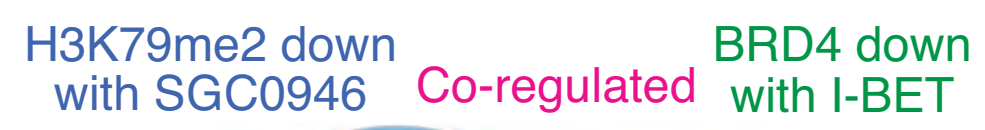

- DMSO

- I-BET

- SGC0946

- I-BET +

SGC0946
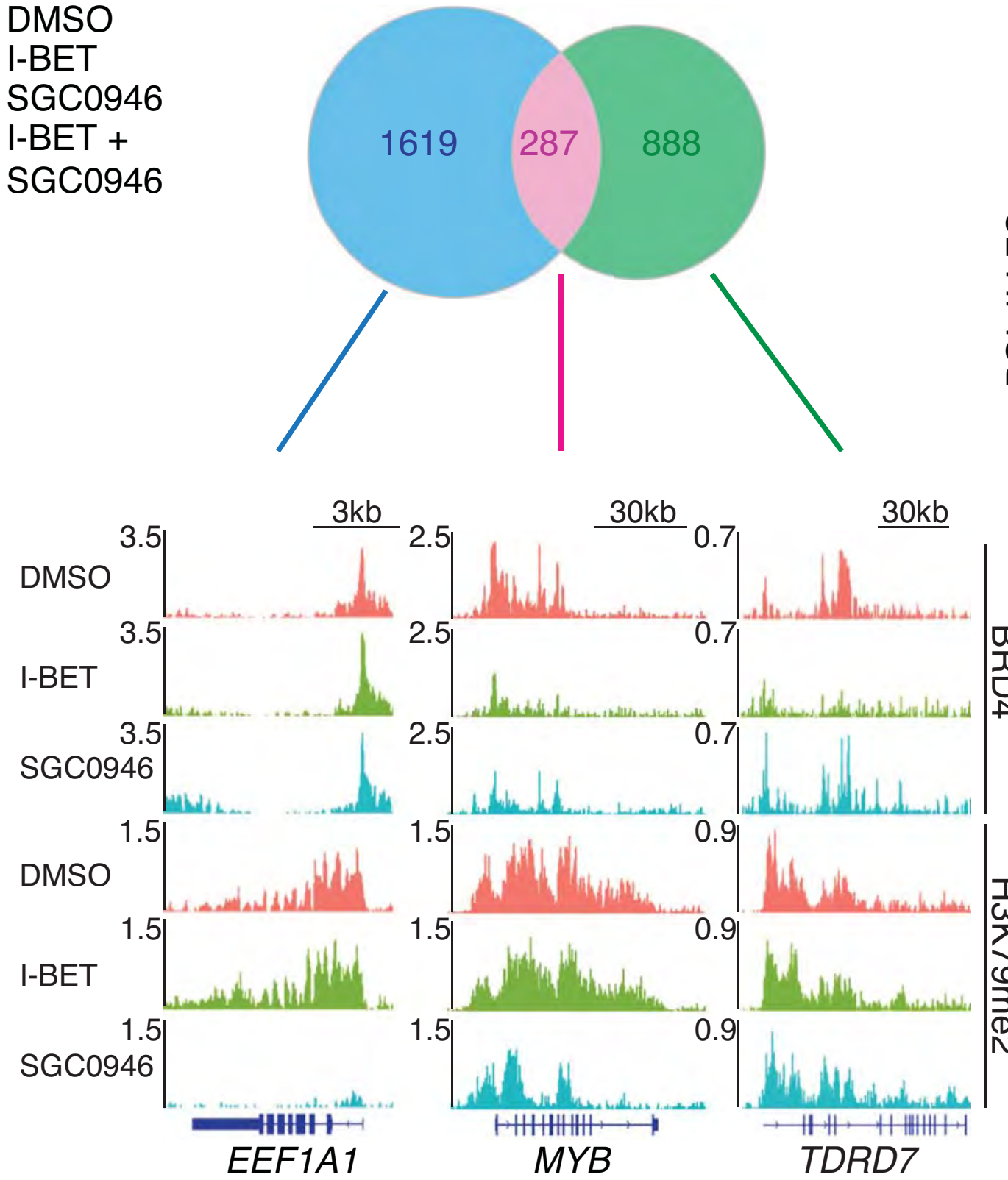

TDRD7

\section{e I-BET treatment}

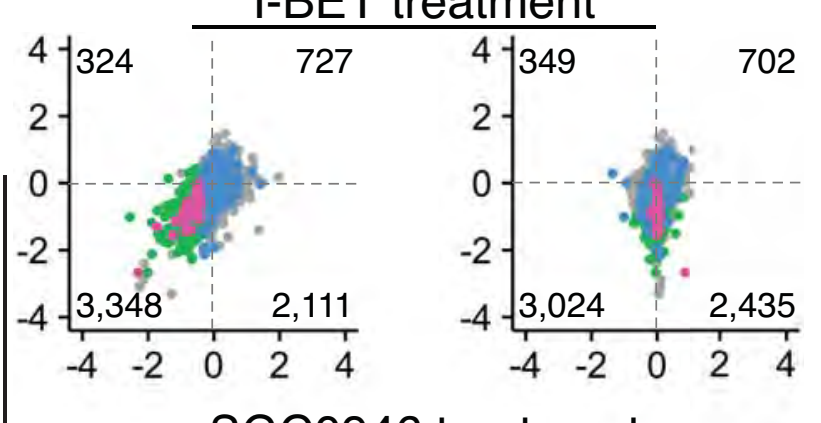

SGC0946 treatment

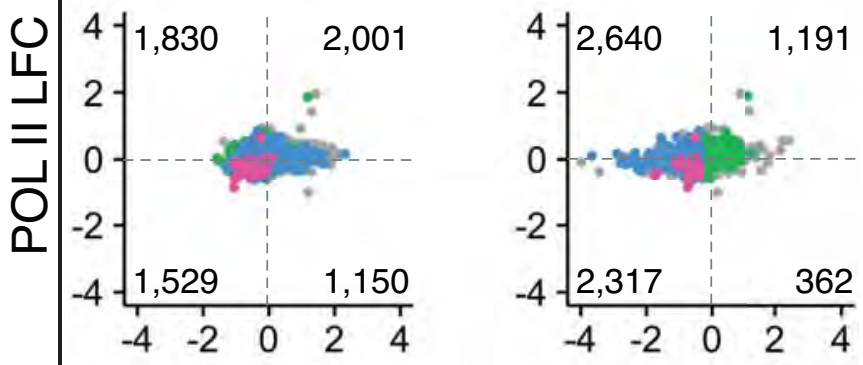

SGC0946 + I-BET treatment
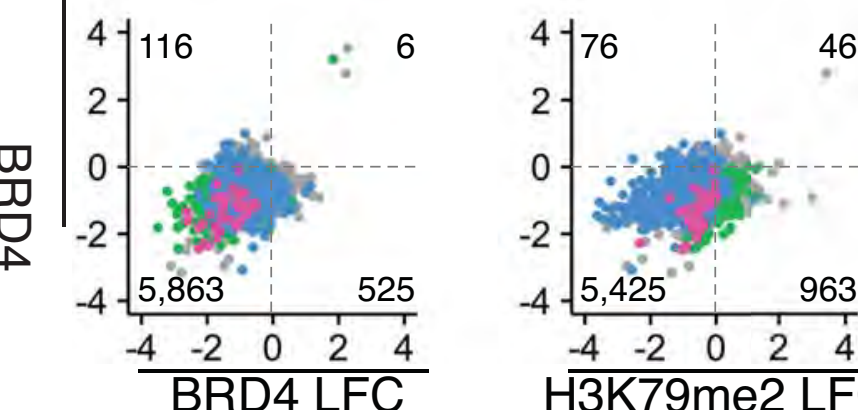

$-2$

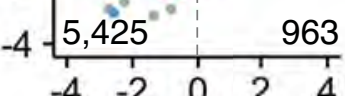
H3K79me2 LFC

- unregulated - SGC0946 regulated

- I-BET regulated co-regulated

$f$
4
$=$
$=$
0
0

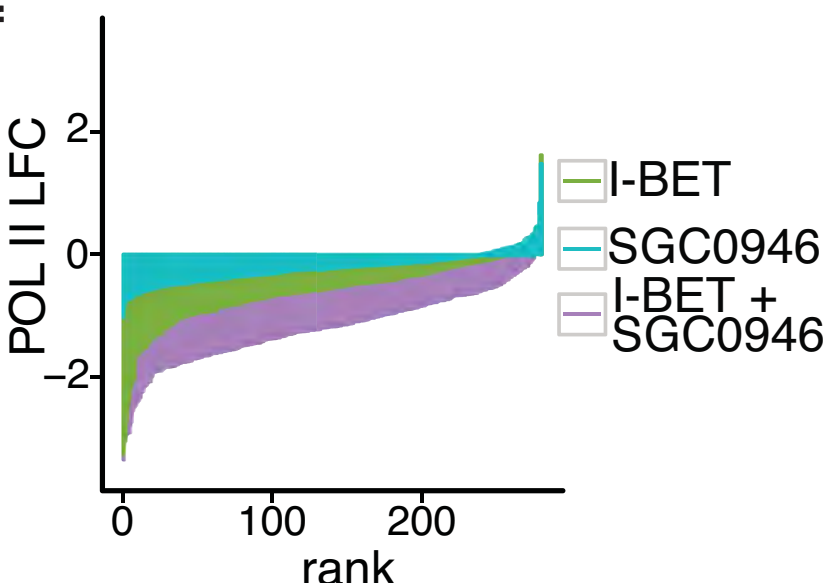


Figure 5

a

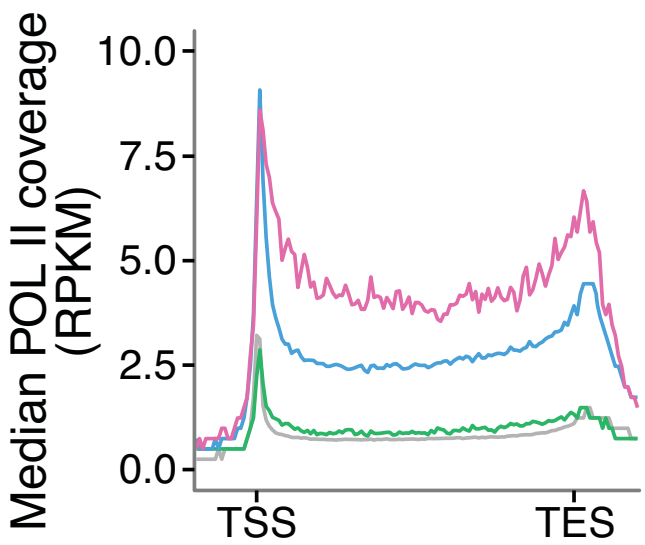
- unregulated $\quad$-I-BET regulated - SGC0946 regulated —co-regulated

C

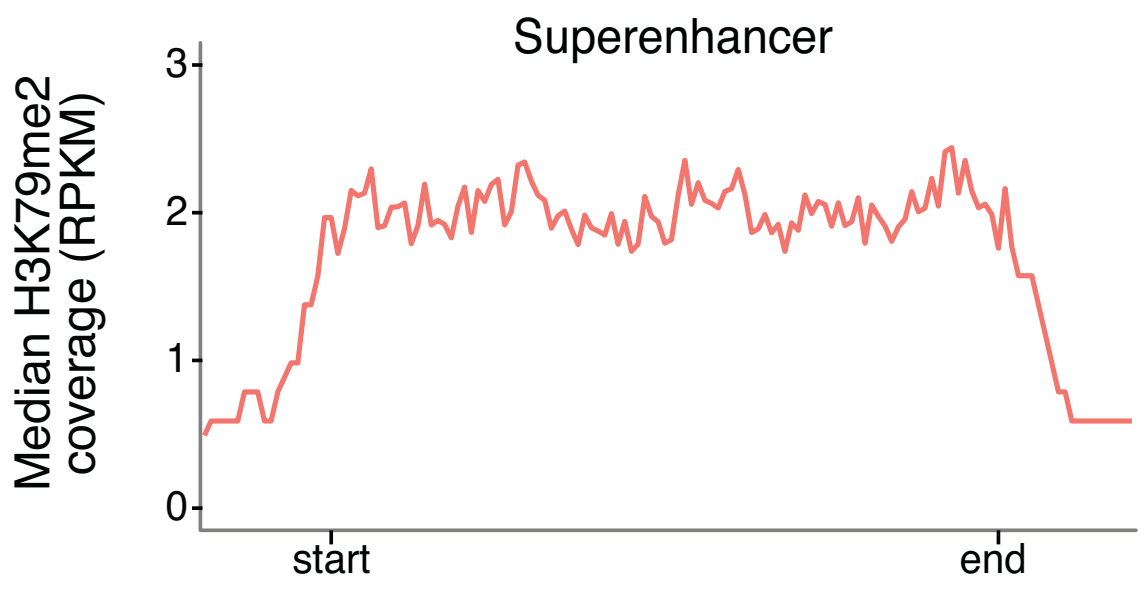

d

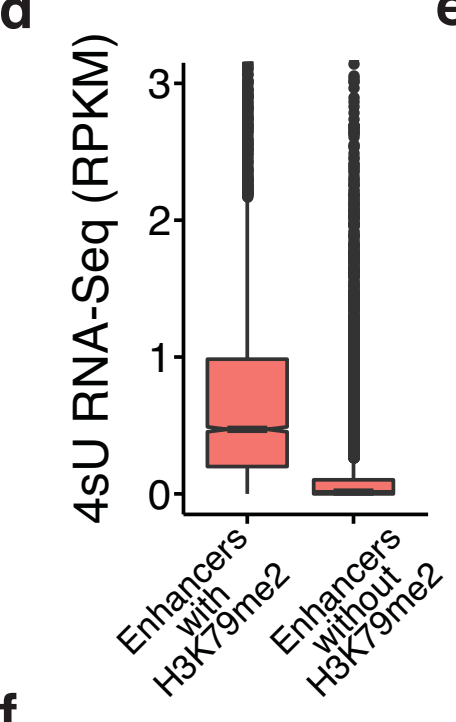

f

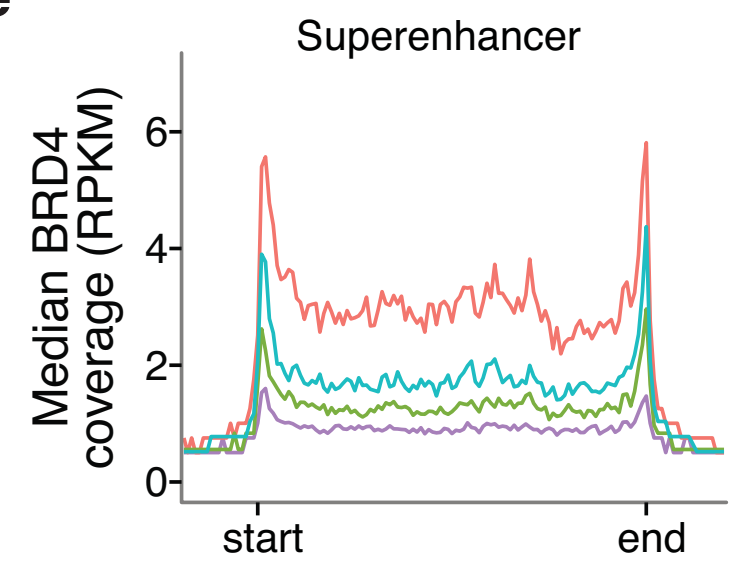

Superenhancer
Typical

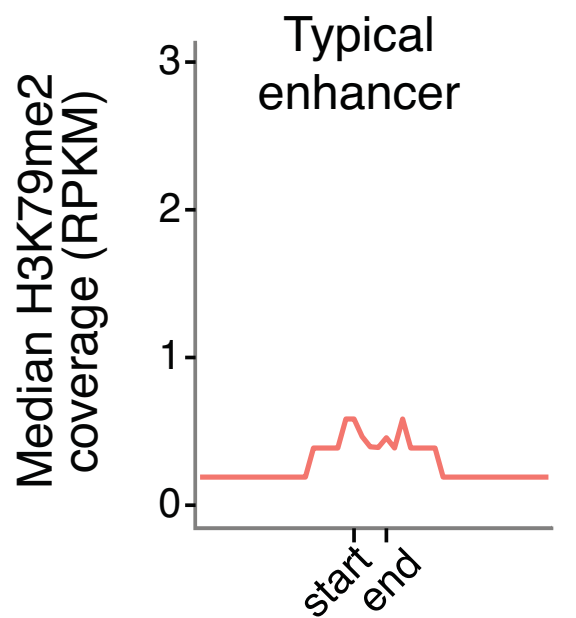

Distance to nearest Superenhancer (kb)

- unregulated $\quad$ - I-BET regulated SGC0946 regulated - co-regulated

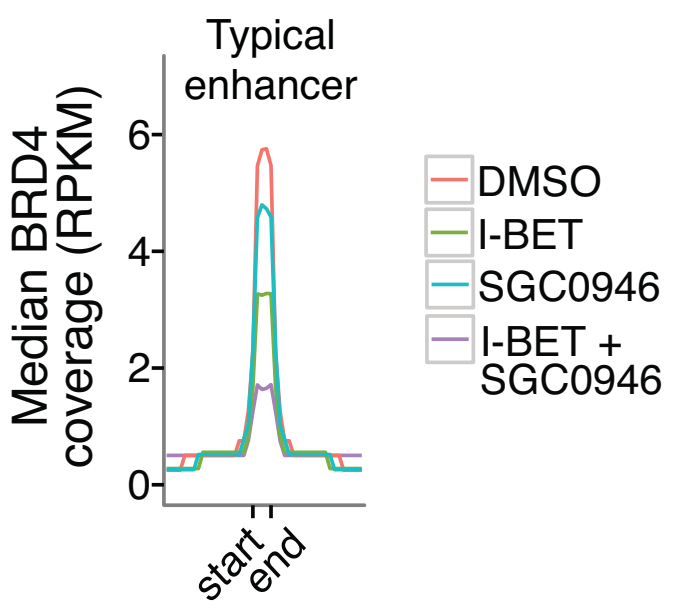

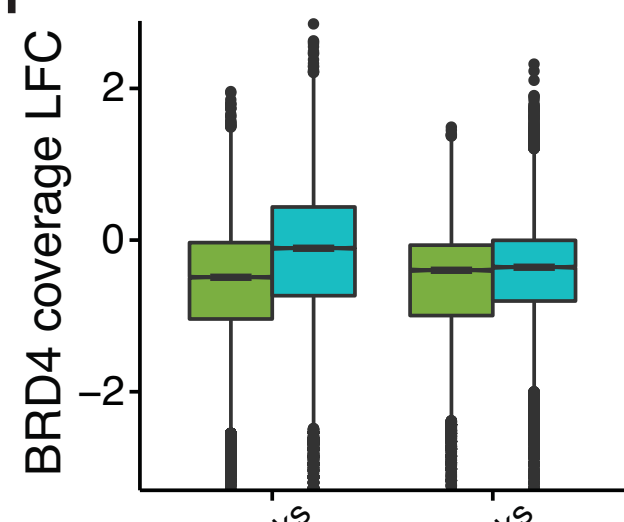

$\partial^{15}$

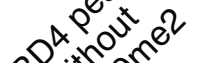
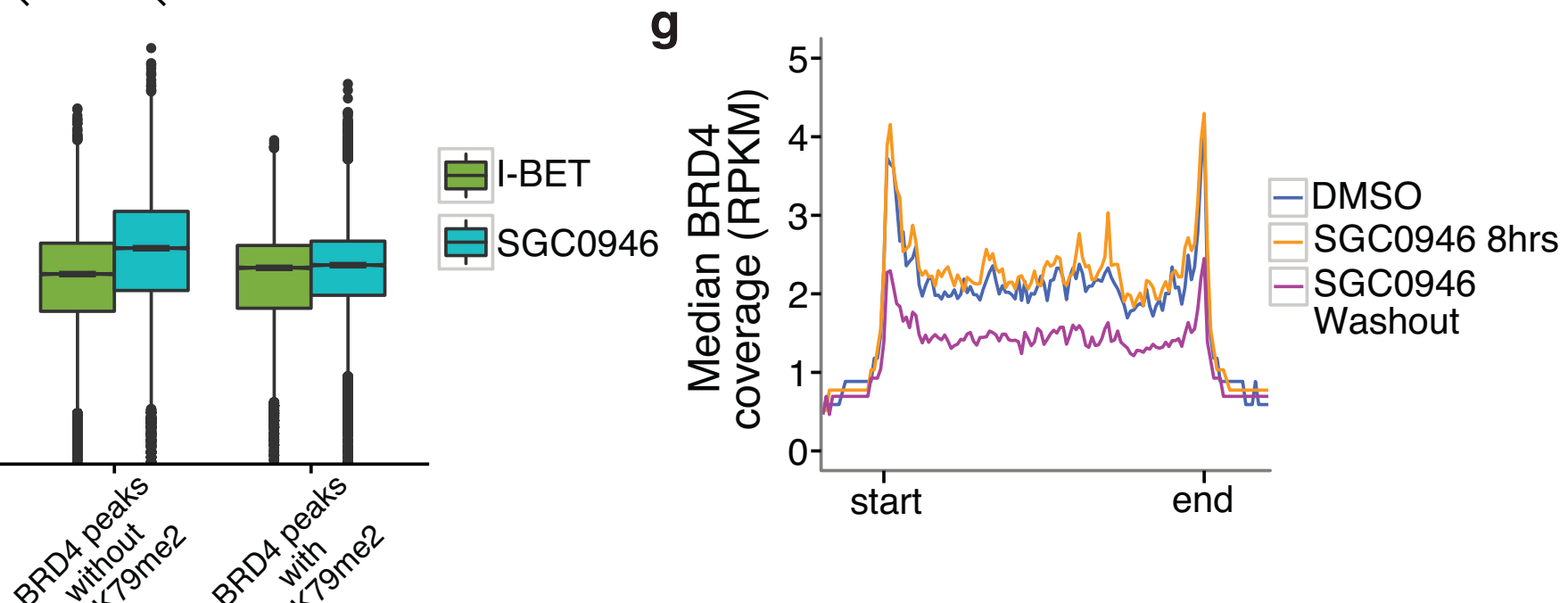


\section{Figure 6}

a
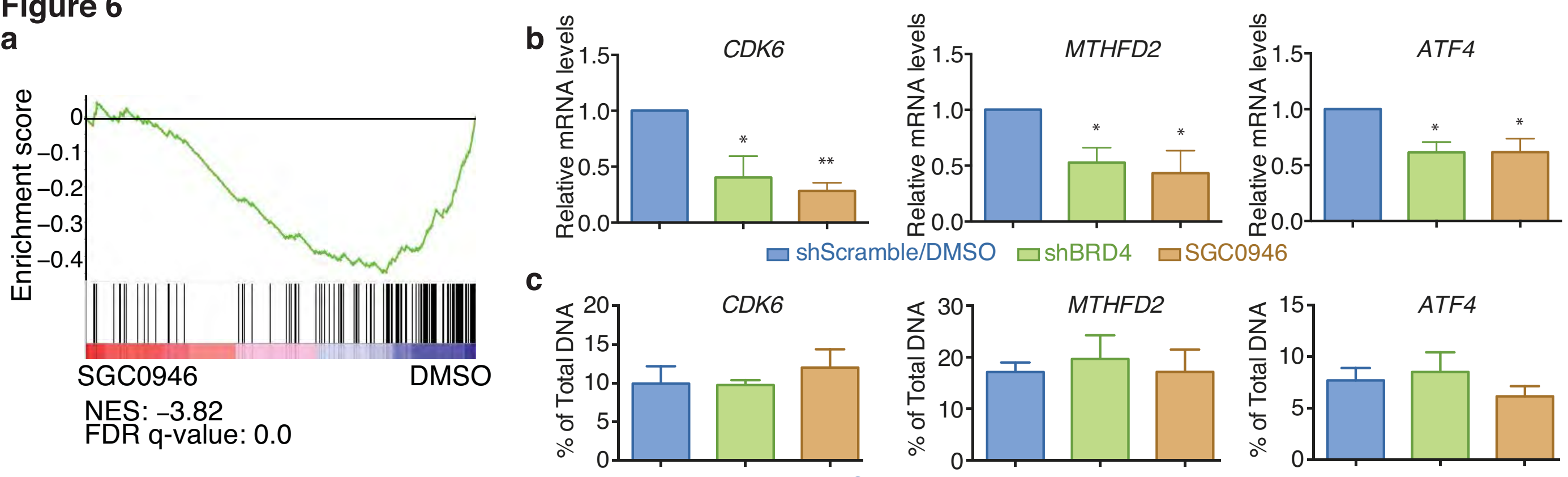

d
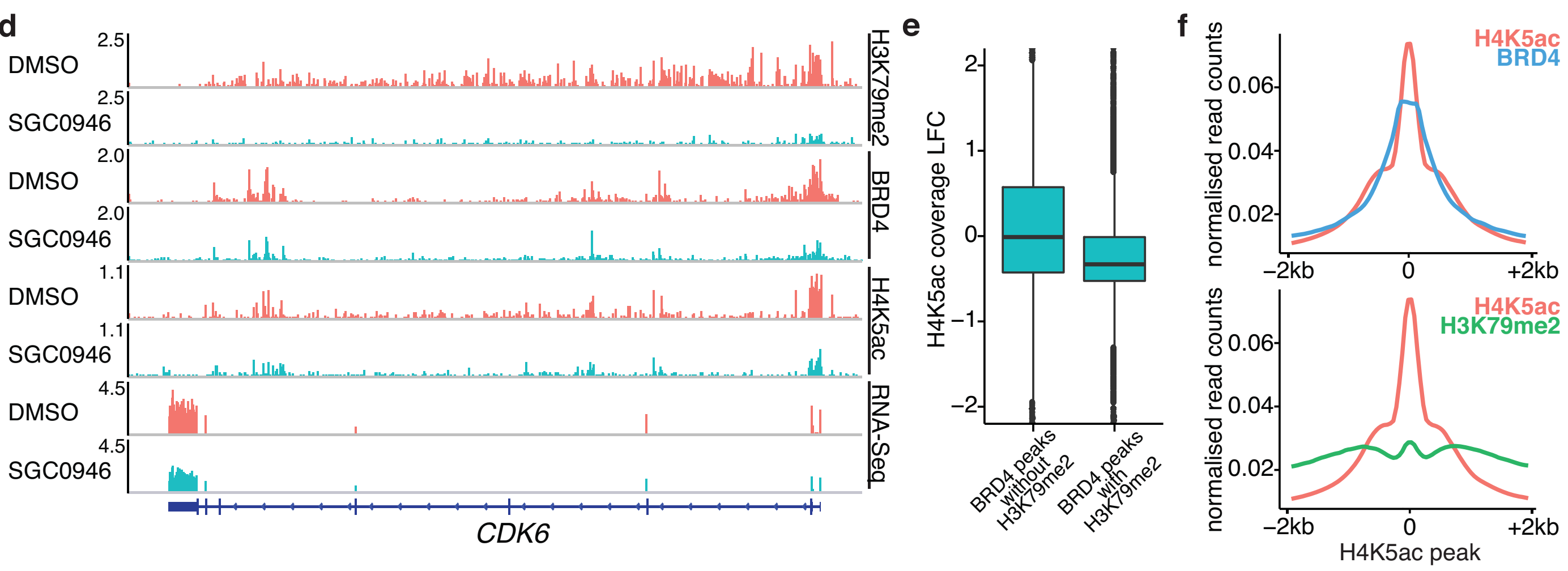


\section{Figure 7}

a
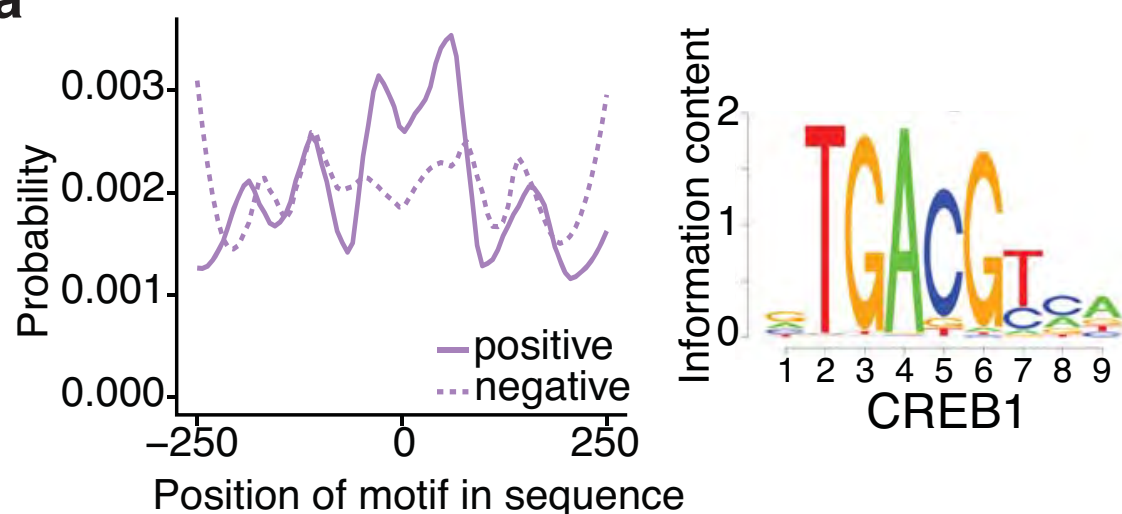

d
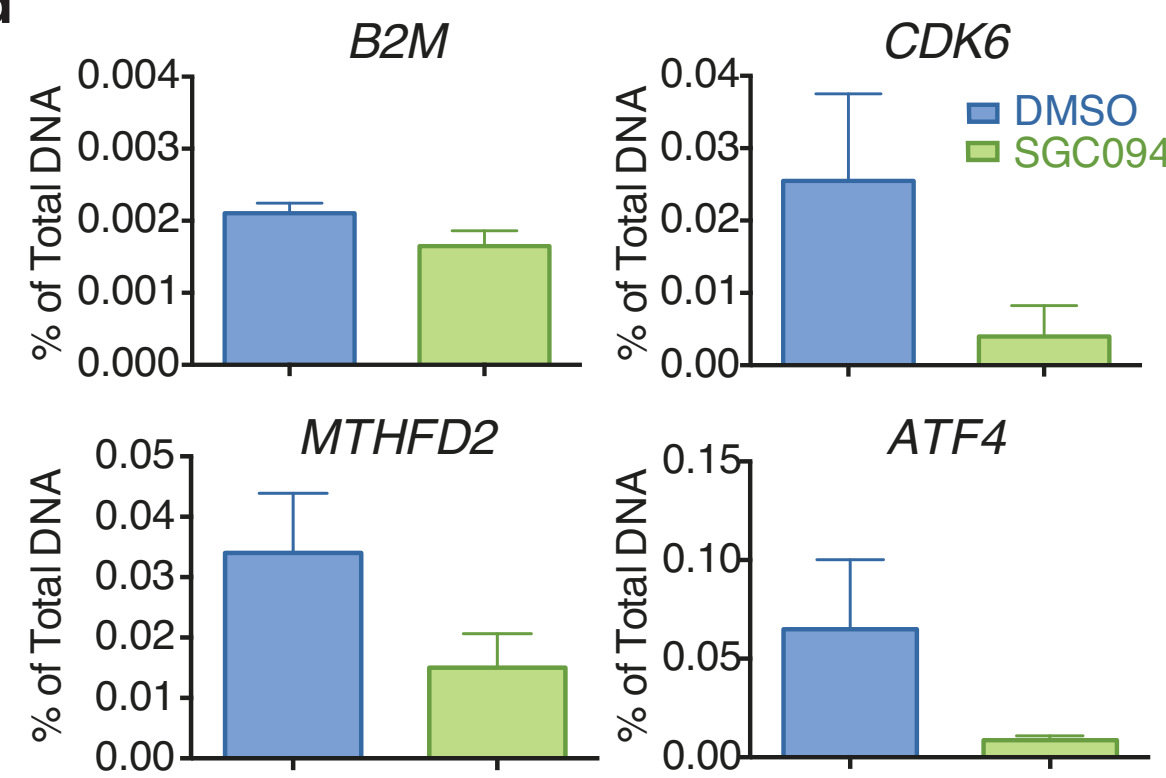

0.00 b
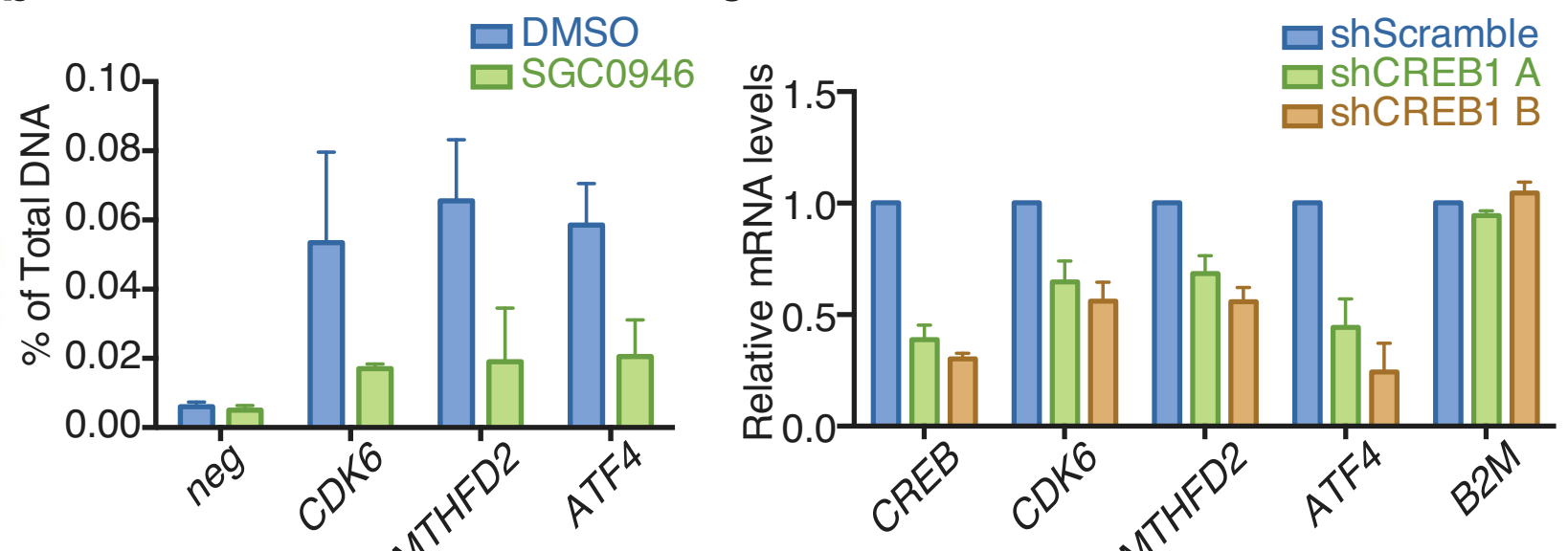

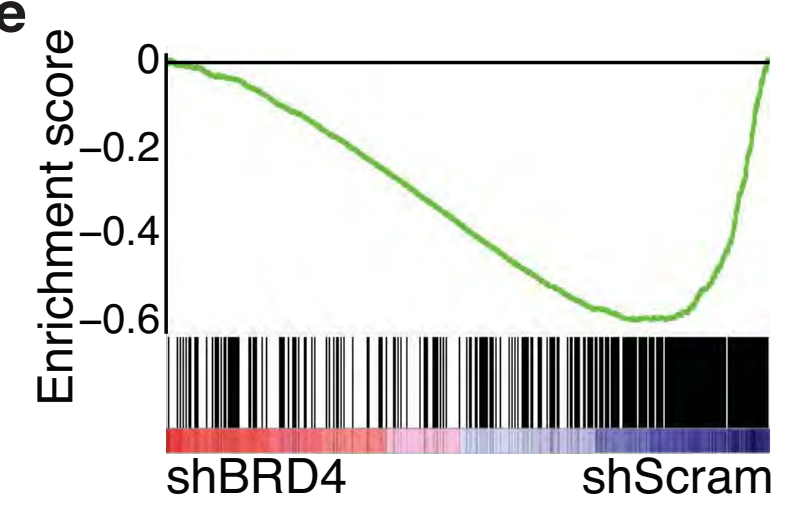

NES: -2.82

FDR q-value: 0.0

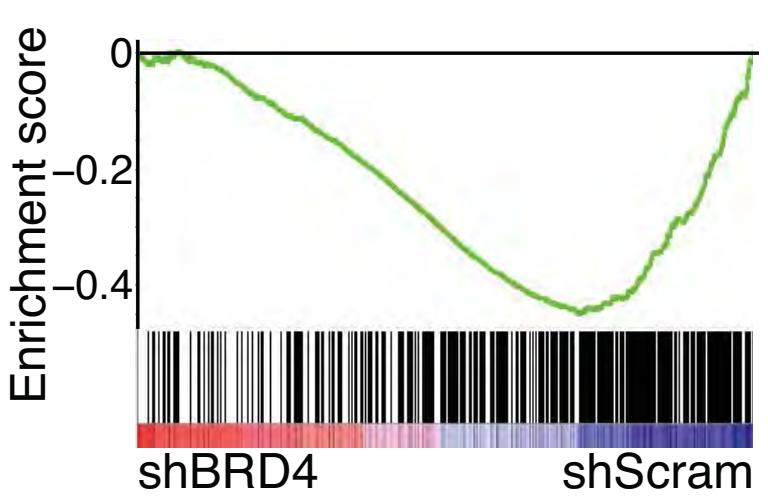

NES: -2.17

FDR q-value: 0.0 
Figure 8

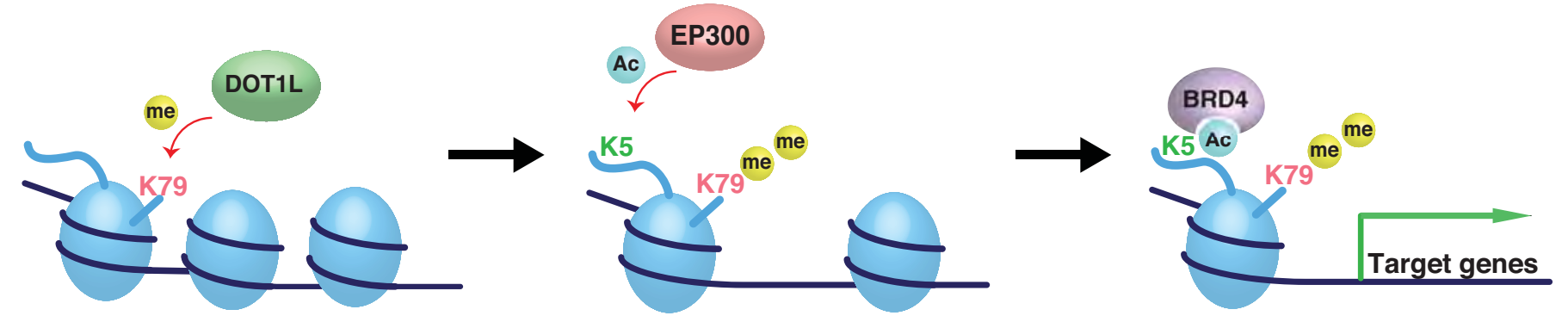




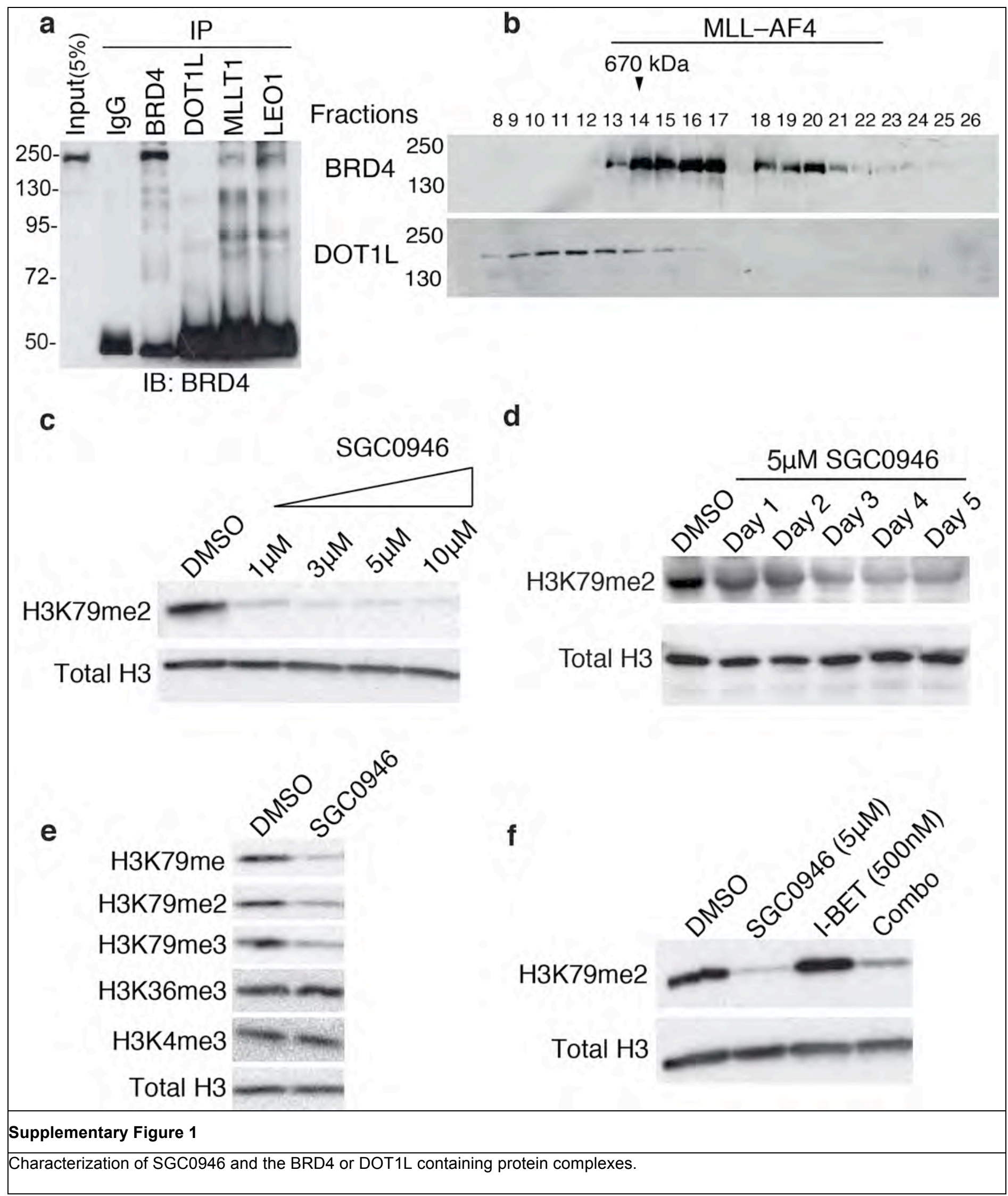


(a) Co-immunoprecipitation (Co-IP) of endogenous BRD4, DOT1L, MLLT1, and LEO1 in MOLM-13 cells. IgG was used as a control. Co-IP was followed by western blot analysis with antibodies against BRD4. (b) Size exclusion chromatography followed by western blot analysis of BRD4 and DOT1L, in MV4;11 cells. (c) Dose response to SGC0946 treatment in MV4;11 cells by western blot analysis using H3K79me2 antibodies, total H3 was used as control. (d) Time course of SGC0946 treatment in MV4;11 cells followed by western blot analysis for H3K79me2. (e) Western blot analysis of various histone lysine methylations (H3K79me/2/3, H3K36me3, and H3K4me3) following SGC0946 treatment in MV4;11 cells. (f) MV4;11 cells were treated with vehicle (DMSO), SGC0946, I-BET or combination followed by western blot analysis with antibodies against H3K79me2 and total $\mathrm{H} 3$. 


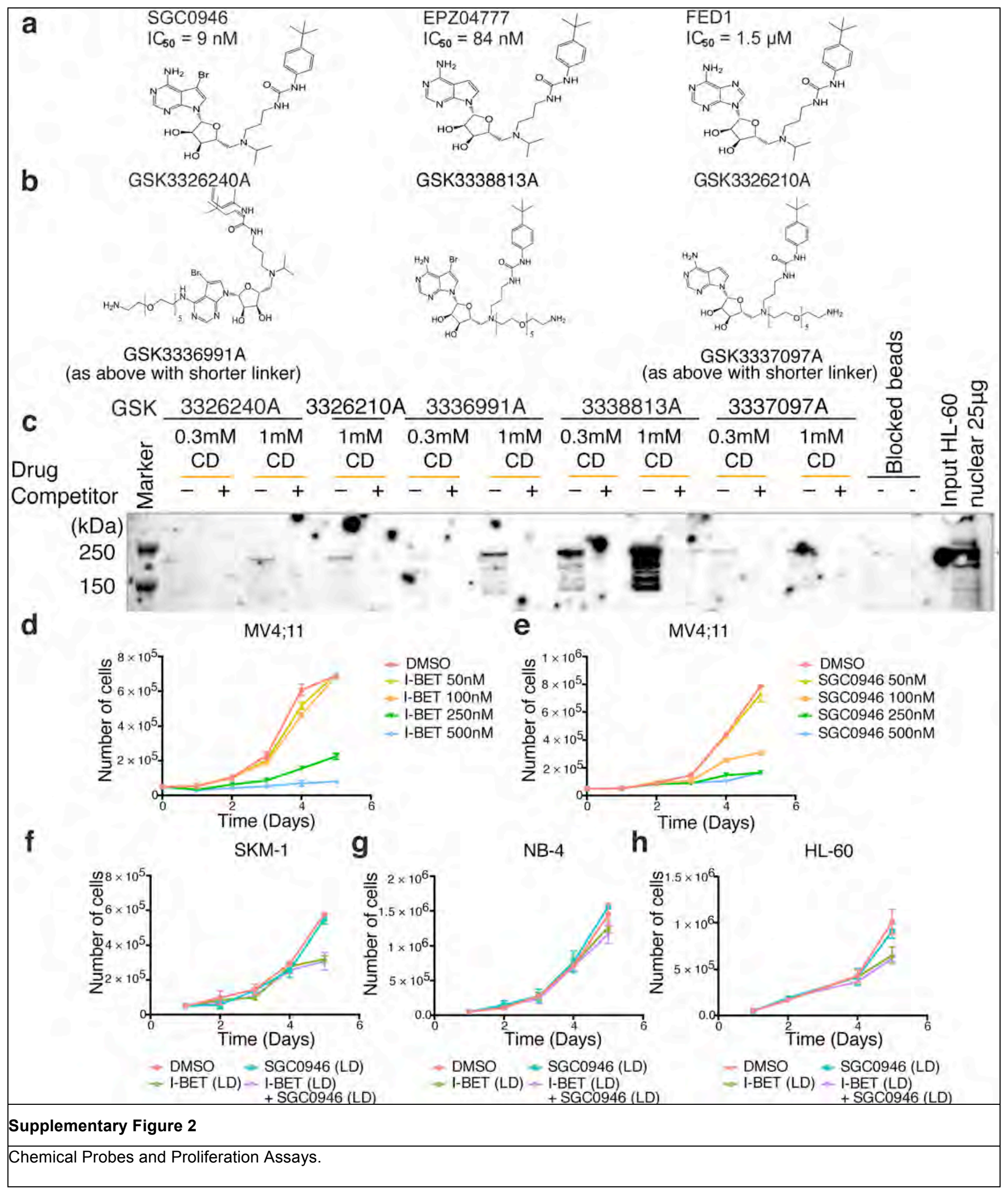


(a) Chemical structure of the DOT1L inhibitors used in this study. (b) Chemical structure of DOT1L inhibitor compounds used for bead immobilization. (c) Pull downs with DOT1L inhibitor compounds immobilized on beads at 2 different coupling densities (CD) in HL60

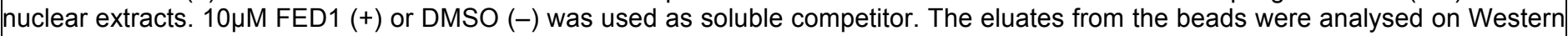
blot using DOT1L antibodies. In vitro sub-therapeutic doses of (d) I-BET and (e) SGC0946 in MV4;11 cells. (f-h) Proliferation assay of the combination of low dose (LD, 100nM) I-BET plus low dose (LD, 1 $\mu \mathrm{M})$ SGC0946 in (f) SKM-1, (g) NB-4 (APML) and (h) HL-60 cells. Mean, error bars, s.d. ( $n=2$ cell culture replicates), representative graph from experiments done on 3 separate occasions. 


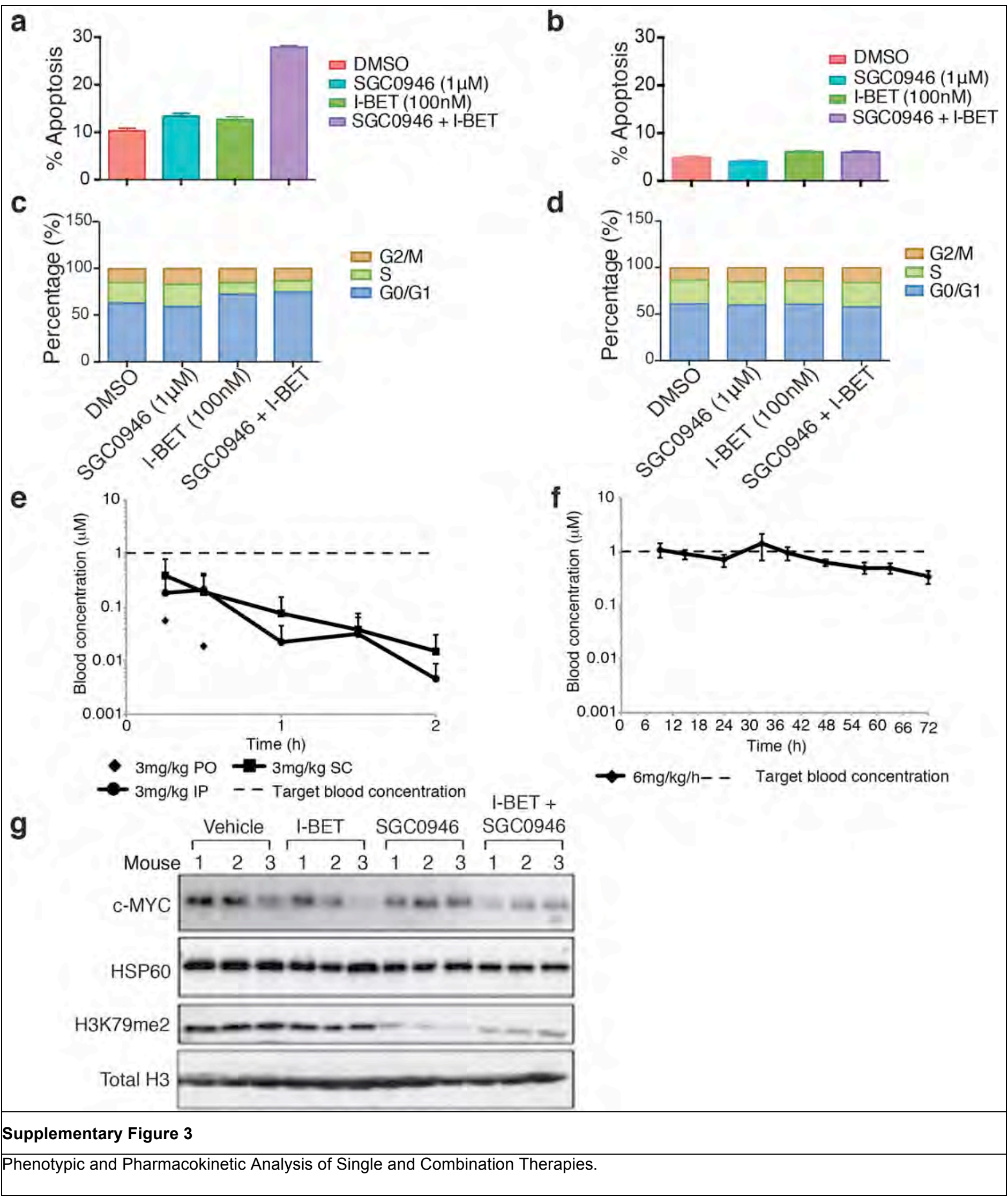


(a-b) Apoptotic response and cell cycle arrest in the combination treated cells. Annexin V staining of (a) MV4;11 and (b) K562 cells treated with either DMSO, SGC0946, I-BET, or combination. Mean, error bars, s.d. ( $\mathrm{n}=3$ cell culture replicates), representative graph from experiments done on 3 separate occasions. (c-d) Cell cycle analysis by PI staining in (c) MV4;11 and (d) K562 cells treated with the inhibitors. These data show a cell cycle arrest in MV4;11 cells that is most prominent with combination therapy. Mean, error bars, s.d. ( $n=3$ cell culture replicates), representative graph from experiments done on 3 separate occasions. (e) Mouse pharmacokinetic studies comparing the blood concentration of SGC0946 administered via PO, SC or IP routes at $3 \mathrm{mg} / \mathrm{kg}$ (Samples taken post $2 \mathrm{~h}$ below limit of quantification; $0.02 \mu \mathrm{M}$ ). Mean, error bars, s.d. ( $n=3$ mice per group). (f) Mouse pharmacokinetic study blood concentrationtime profile of SGC0946 administered via surgically implanted mini-pump targeting $6 \mathrm{mg} / \mathrm{kg} / \mathrm{h}$ for $72 \mathrm{~h}$. Required exposure for in vivo model achieved. Mean, error bars, s.d. ( $\mathrm{n}=3$ mice per group). ( $(\mathbf{g})$ Western blot analysis of in vivo on-target activity of I-BET and SGC0946 on the bone marrows of three mice per group, using c-MYC, HSP60, H3K79me2 and total H3 antibodies. 


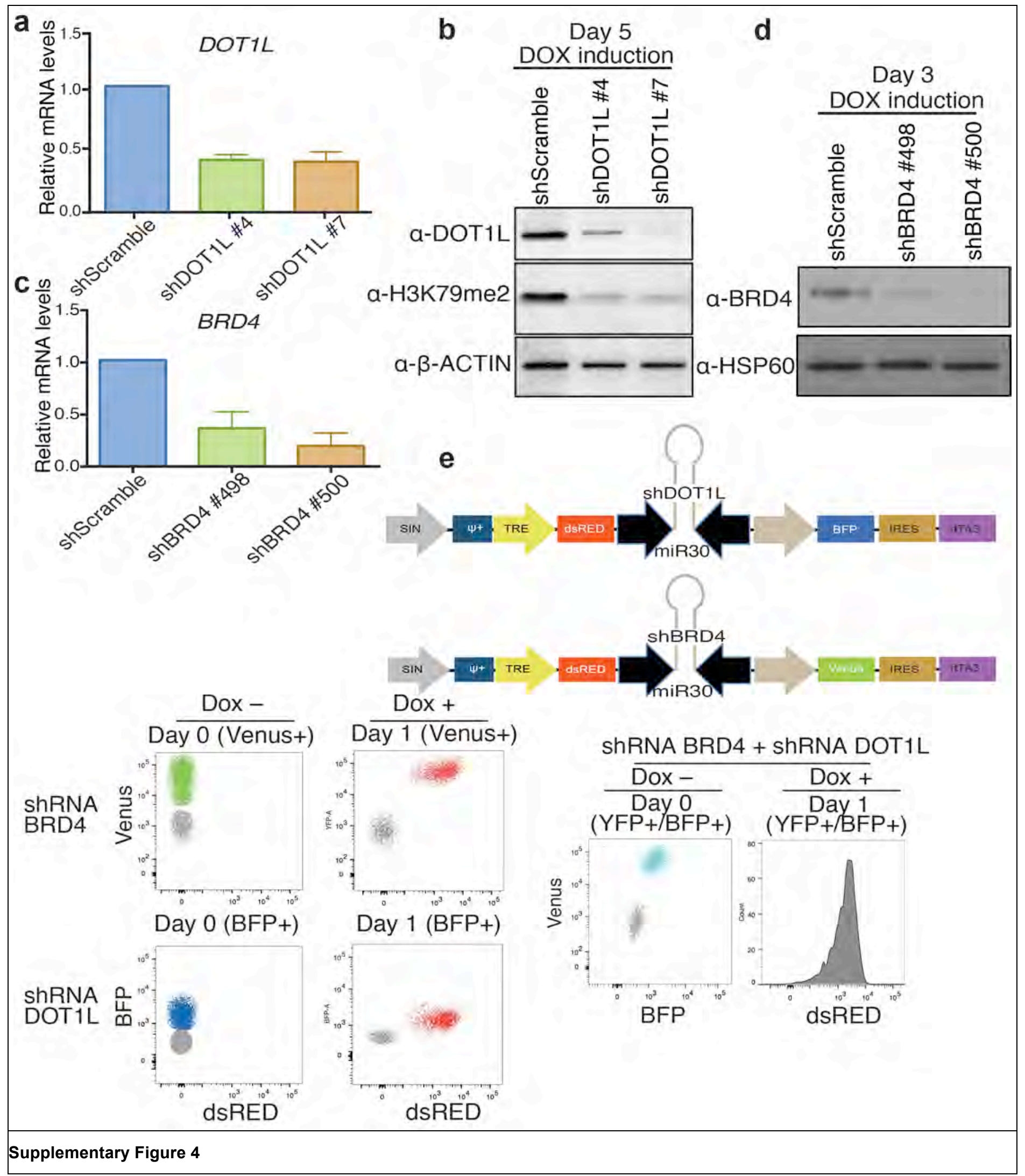


Inducible RNAi Knock-down of BRD4 and DOT1L.

(a) Western blot and (b) qRT-PCR analysis of DOT1L expression in inducible RNAi samples. $\beta$-ACTIN was used as a loading control (c) Western blot and (d) qRT-PCR analysis of BRD4 expression in inducible RNAi samples. HSP60 was used as a loading control. shDOT1L \#7 and shBRD4 \#498 were used in the competition assays (Figure 3c). (e) Schematic overview of the plasmids used. Flow plot examples showing cells transduced with single and combination inducible RNAi constructs expressing two different fluorophores. This strategy allowed for double knockdown within the same cell. 


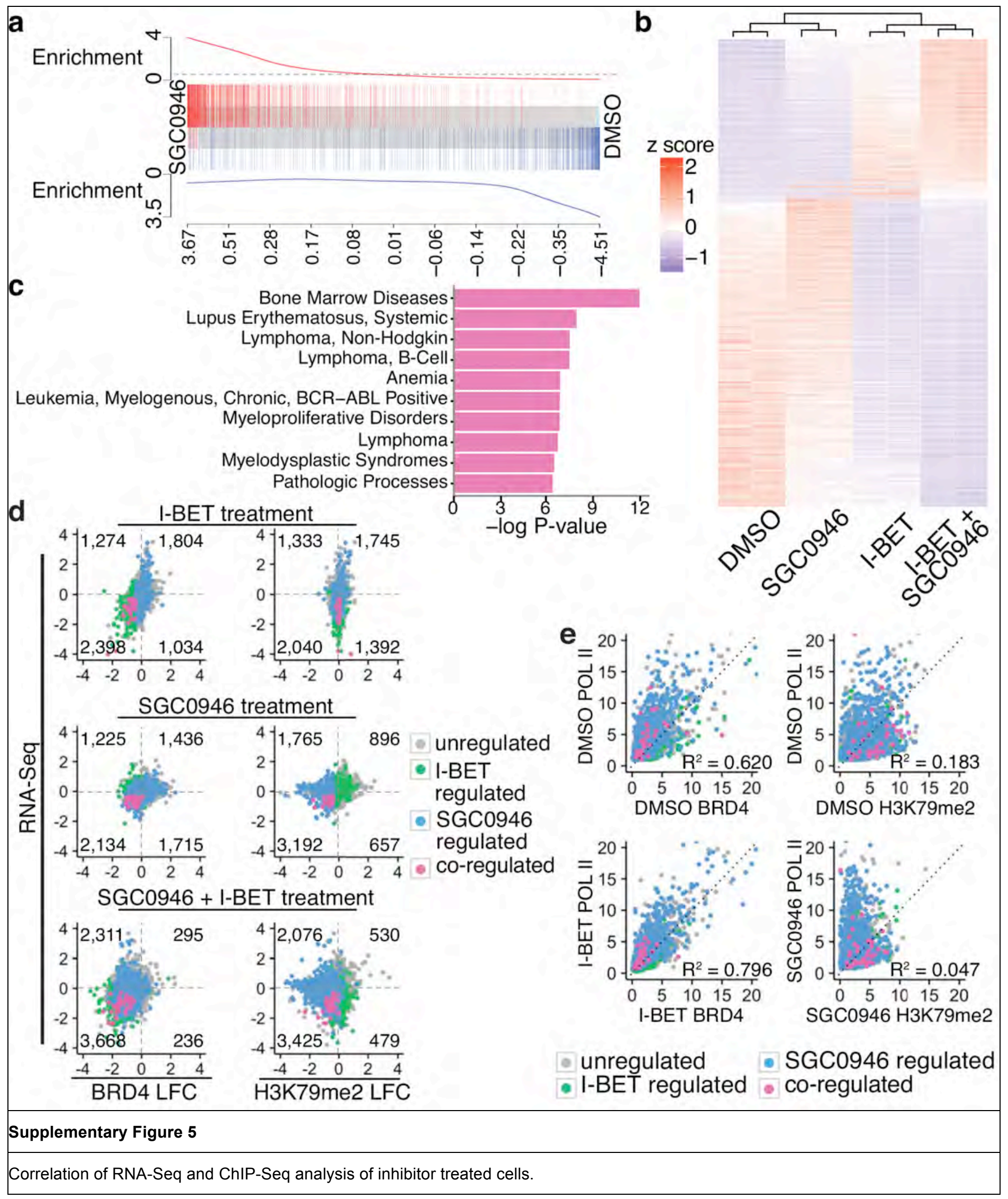


(a) GSEA using ROAST (Wu, D. et al. Bioinformatics 26, 2176-2182, 2010) of differentially expressed genes from RNA-Seq data of SGC0946 treated cells with previously published up- (red) and down-regulated (blue) genes from microarray analysis of MV4;11 cells treated with EPZ004777 (GSE29828). The shaded area in the centre of the plot shows genes ranked by log fold change in expression in SGC0946 compared with DMSO treated cells. Pink and blue shading represent significantly up- and down- regulated genes, respectively. (b) Heatmap of differential mRNA expression data from RNA-Seq of MV4;11 cells treated with I-BET, SGC0946, or combination in duplicate cell culture experiments. (c) geneGO analysis of the top ten diseases associated with the co-regulated genes. (d) Change in gene expression from RNA-Seq following BRD4 inhibition (I-BET), DOT1L inhibition (SGC0946) or a combination of BRD4 and DOT1L inhibition plotted against the changes in BRD4 binding and H3K79me2 levels in genes from ChIP-Seq. (e) Correlation between POL II and BRD4 binding or H3K79me2 levels at all genes in DMSO, I-BET and SGC0946 treated cells. 


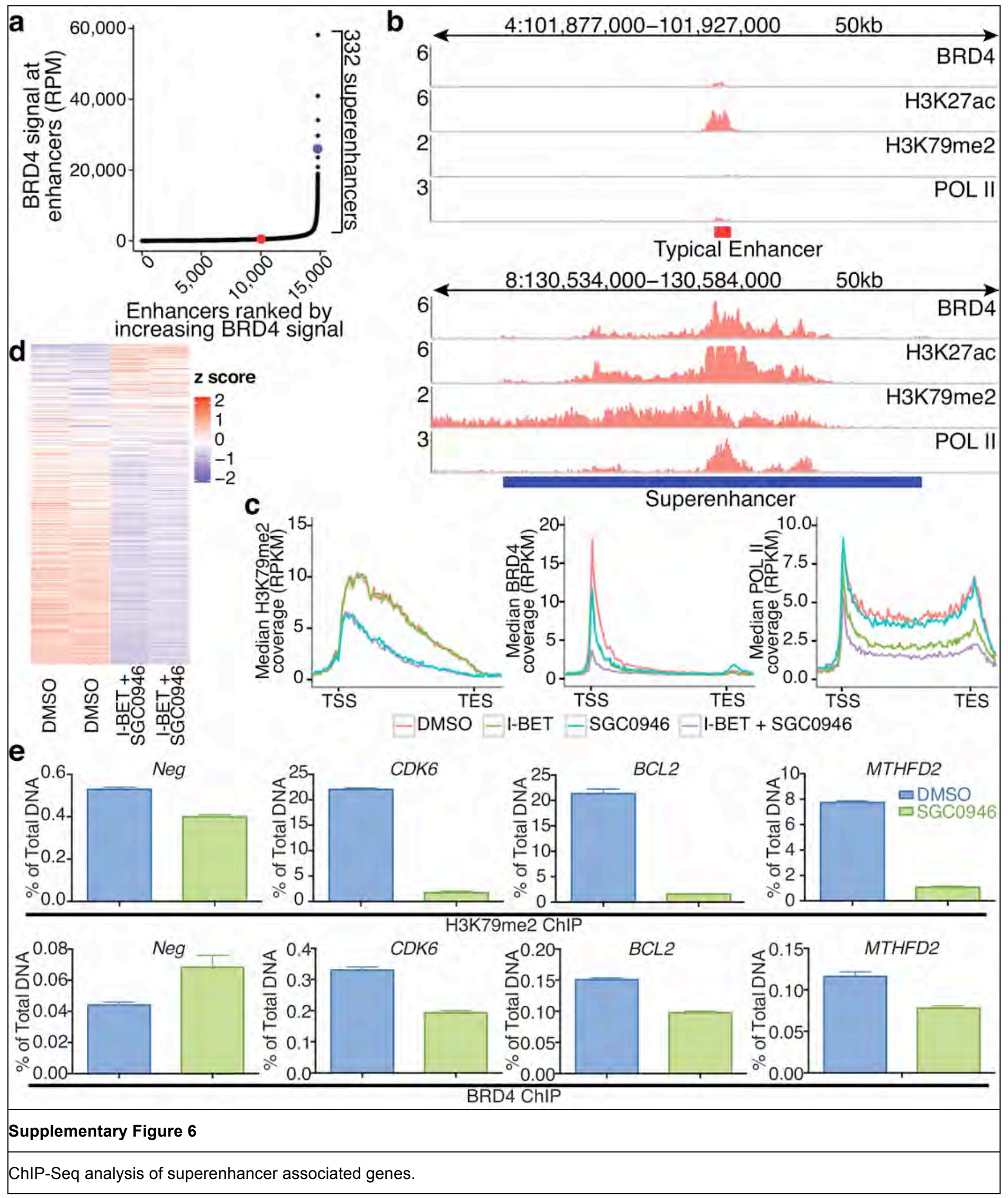


(a) Enhancers ranked by increasing BRD4 ChIP-Seq signal in reads per million (RPM) mapped reads. (b) ChIP-Seq profile of BRD4 H3K27ac, H3K79me2 and POL II at a typical enhancer and superenhancer indicated in Supplementary Fig 6a, in red and blue respectively. (c) Median H3K79me2, BRD4 and POL II coverage in RPKM across the co-regulated genes following I-BET, SGC0946 or combination treatment. (d) Heatmap of the expression of genes in closest proximity to superenhancers with I-BET and SGC0946 combination treatment. (e) qPCR analysis of H3K79me2 and BRD4 ChIP in MOLM-13 cells treated with SGC0946 using primers targeting CDK6, BCL2, MTHFD2 and Neg (negative control region). Mean, error bars, s.d. ( $\mathrm{n}=3$ technical replicates), representative graph from experiments done on 3 separate occasions 


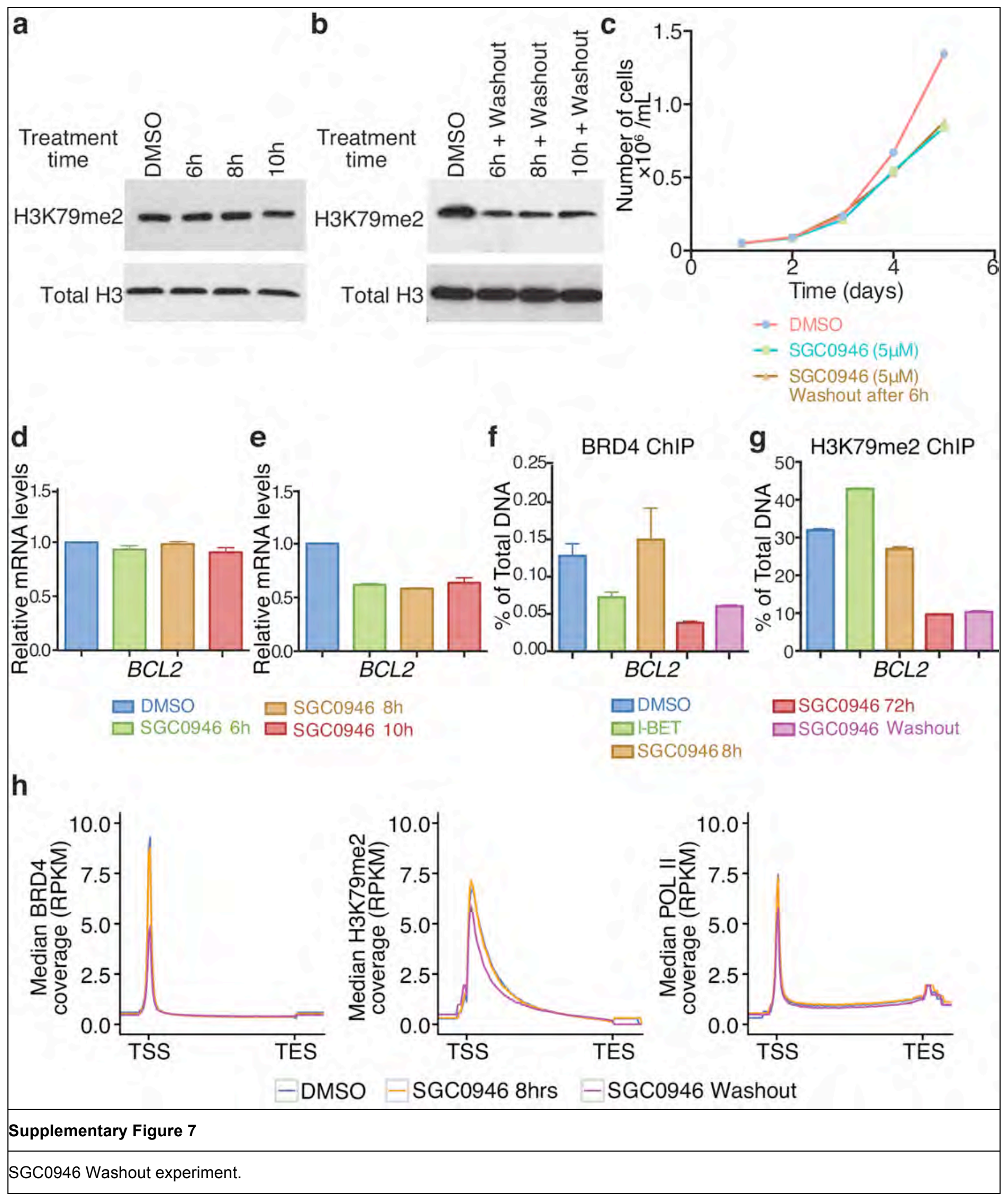


(a-b) Anti-H3K79me2 and anti-total H3 western blot. Samples are lysates from MV4;11 cells treated with DMSO or SGC0946 for 6, 8 and $10 \mathrm{~h}$ and (a) harvested at end of treatment or (b) after washout of SGC0946, then harvested 72 hours later. (c) Proliferation Assay. Cell counts after short term treatment of MV4;11 cells with SGC0946 for 6 hours followed by wash-out or continuous treatment with SGC0946. (d-e) qRT-PCR of BCL2 expression from MV4;11 cells treated with DMSO or SGC0946 for 6, 8 and $10 \mathrm{~h}$ and (d) harvested at end of treatment or (e) after washout of SGC0946, then harvested 72 hours later. ChIP-qPCR analysis for (f) BRD4 and (g) H3K79me2. MV4;11 cells were treated with I-BET for 6 hours, SGC0946 for 8 hours, SGC0946 for 72 hours continuously or SGC0946 for 8 hours followed by washout and assessed 72 hours later. Mean, error bars, s.d. ( $\mathrm{n}=3$ technical replicates), representative graph from experiments done on 3 separate occasions. (h), Median BRD4, H3K79me2 and POL II levels in RPKM across genes containing both BRD4 and H3K79me2 including 5kb upstream and downstream regions following short-term SGC0946 or SGC0946 washout treatment. 


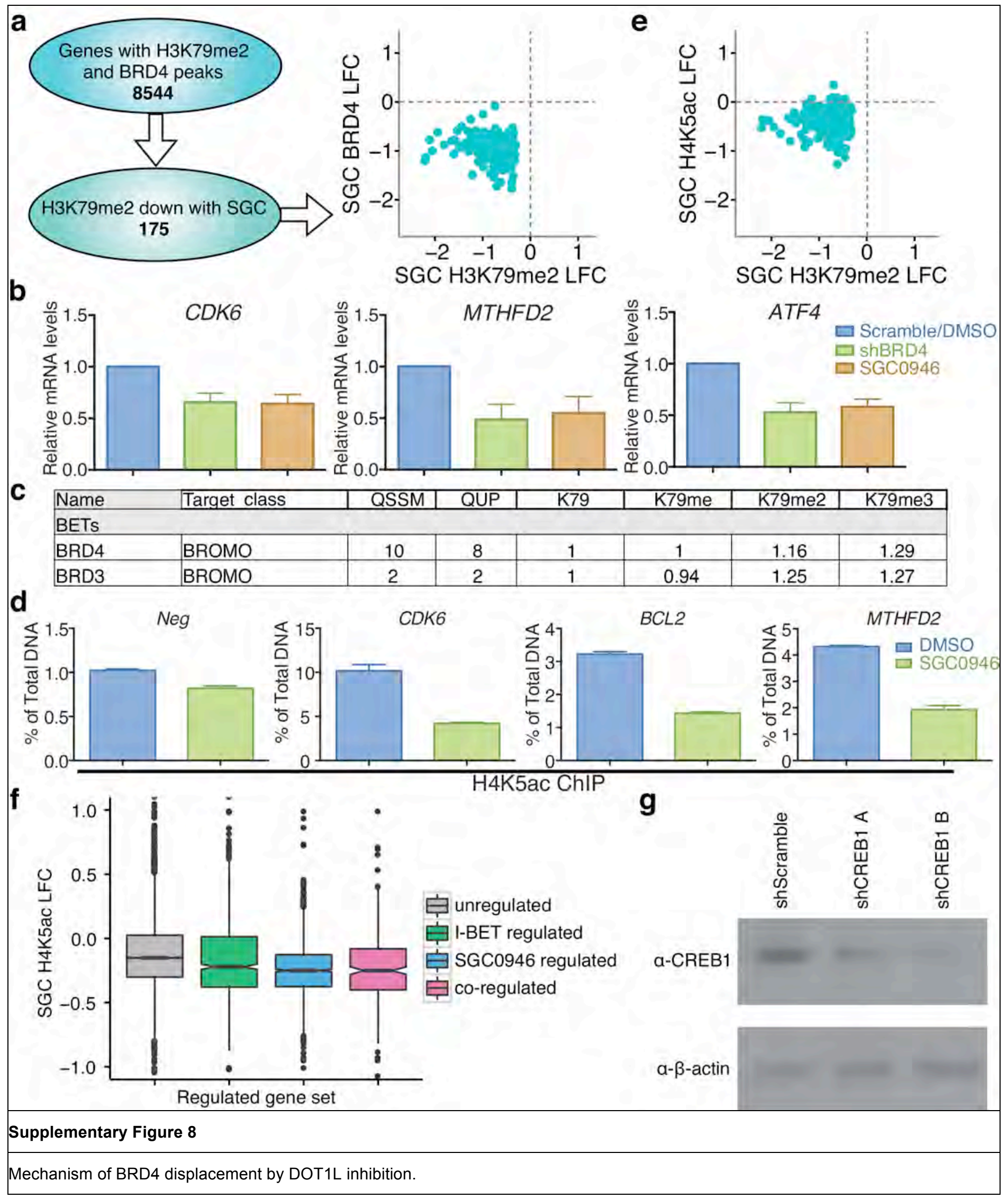


(a) Left, Flow chart hierarchy of the numbers of expressed genes with H3K79me2 and BRD4 peaks that change their expression and have decreased H379me2 levels after SGC0946 treatment. Right, scatterplot of the log-fold change in BRD4 and H3K79me2 after SGC0946 treatment in the 175 SGC0946 regulated genes. (b) qRT-PCR of genes identified by RNA-seq as being regulated by both BRD4 depletion and DOT1L inhibition. Mean, error bars, s.d. ( $n=3$ experiments done on 3 separate occasions). (c) Quantitative proteomic analysis of BRD3 and BRD4 within HL-60 nuclear extracts that are bound to various biotinylated histone H3K79 peptides. BRD3 and BRD4 captured by the histone peptides were differentially quantified by isobaric tagging, as previously performed (Dawson, M.A. et al. Nature 478, 529-33, 2011). (d) ChIP-qPCR analysis of SGC0946 treated MOLM-13 cells using primers against CDK6, $B C L 2, M T H F D 2$ and Neg (Negative control region). Mean, error bars, s.d. ( $n=3$ technical replicates), representative graph from 3 experiments done on 3 separate occasions. (e) Scatterplot of the log-fold change in H3K79me2 and H4K5ac levels after DOT1L inhibition in the 175 SGC0946 regulated genes. (f) Log-fold change in the levels of H4K5ac in the subsets of I-BET regulated, SGC0946 regulated and co-regulated genes following SGC0946 treatment. The upper limit, center and lower limit of boxplots denote the upper quartile, median and lower quartile of the data, respectively. Whiskers extend to $1.5 \times$ interquartile range above and below the upper and lower quartiles, respectively. (g) Western blot of CREB1. Lysate from MV4;11 cells transduced with the two independent shRNAs used in Figure 7. 